\title{
LA FORMAZIONE DEI PARTITI CONFESSIONALI IN EUROPA
}

\author{
di Stathis N. Kalyvas
}

Di recente, la ricerca sociologica per lo più quantitativa ha riaffermato l'importanza della religione riguardo all'azione politica e alle politiche pubbliche. Tra i suoi contributi vi sono vari modelli di associazione tra la religione, nella sua variante cattolica, ed una pluralità di esiti politici e di policy. Castles (1994) ha individuato un'evidenza prima facie per cui il cattolicesimo è associato con molti esiti di policy quali la spesa assistenziale, le politiche della famiglia, ed esiti connessi al mercato del lavoro. Misra e Hicks (1994) hanno trovato una relazione positiva tra ciò che essi chiamano «cultura romano-cattolica» e livello di sindacalizzazione. Infine, Wilensky (1981), Esping-Andersen (1990), Huber, Ragin e Stephens (1993), e più recentemente Van Kersbergen (1995) hanno tutti rilevato che i partiti cristiano-democratici sono correlati ad alti livelli di spese sociali. Queste scoperte sono ancor più significative se si considera che sono state fatte nel contesto delle società probabilmente più secolarizzate: quelle dell'Europa occidentale contemporanea. Proprio per questo, gli autori di tali studi sottolineano la portata più ampia delle loro scoperte e reclamano una rinnovata attenzione alla religione, al cattolicesimo, ai partiti cristiano-democratici, affermando che «per gli anni ' 90 il cattolicesimo dovrebbe fornire una prospettiva fruttuosa per lo studio della political economy così come per gli anni '80 la socialdemocrazia» (Misra e Hicks 1994, 319).

La religione entra in questi studi per due vie. Una è quella demografica: l'ampiezza della popolazione cattolica (Castles 1994; Misra e Hicks 1994). L'altra è politica e istituzionale: l'in-

Per una trattazione più completa dell'argomento sviluppato in questo articolo si veda il mio libro, in corso di pubblicazione From Pulpit to Party. The Rise of Christian Democracy in Europe, Ithaca-London, Cornell University Press, 1996.

RIVISTA ITALIANA DI SCIENZA POLITICA / a. XXVI, n. 2, agosto 1996 
cumbency dei partiti cristiano-democratici (Castles 1994; Huber, Ragin e Stephens 1993; Misra e Hicks 1994). Mentre l'impiego di variabili demografiche è generalmente considerato molto problematico, l'uso dell'incumbency dei partiti cristiano-democratici è ampiamente accettato a ragione dell'assunto che tali partiti sono «istituzioni cattoliche», «veicoli del "vangelo sociale" e dell'accomodamento della Chiesa alla modernità»' (Misra e Hicks 1994, 305 e 316), e «vie di influenza» della Chiesa cattolica (Castles 1994, 24). In sostanza, si sostiene che questi partiti rappresentino la dottrina sociale cattolica (Huber, Ragin e Stephens 1993, 717) o la «cultura romano-cattolica» (Misra e Hicks 1994, 304). Non sorprende, pertanto, che la menzione dei partiti cristiano-democratici sia tipicamente accompagnata dal riferimento ad encicliche papali quali la Rerum Novarum o la Quadragesimo Anno (Misra e Hicks 1994, 307; Castles 1994, 22; Huber, Ragin e Stephens 1993, 717).

La fusione, largamente condivisa, tra i partiti cristiano-democratici e la Chiesa e la cultura cattoliche è, tuttavia, fuorviante, poiché la letteratura sulla quale si basa è carente e ateorica. $\mathrm{Ne}$ consegue una comprensione fallace della principale variabile dei recenti studi, la democrazia cristiana, e la distorsione delle loro scoperte. L'obbiettivo di questo articolo è duplice. In primo luogo, esso offre una spiegazione nuova della formazione dei partiti confessionali precedenti alla seconda guerra mondiale, i predecessori degli attuali partiti cristiano-democratici, e quindi contribuisce a meglio comprendere le recenti scoperte sociologiche. In secondo luogo, presenta una spiegazione della formazione di quei partiti che è allo stesso tempo analitica, comparata e storica. Tale spiegazione attinge alla nota teoria dei cleavages formulata da Stein Rokkan (1970; si veda anche Lipset e Rokkan 1967) e la rivisita mediante l'applicazione della teoria della scelta razionale. Questo articolo suggerisce che gli approcci fondati esclusivamente sulle associazioni statistiche tra variabili sono fuorvianti, perché mancano di un'analisi storica accurata e validamente argomentata dei meccanismi causali sottostanti. Al contrario, questo articolo mostra che la teoria della

1 Accanto ai problemi di operazionalizzazione e di causazione che comporta (Therborn, 1994), l'ampiezza della popolazione cattolica non costituisce un'adeguata misura della forza dei sentimenti religiosi popolari. La frequenza ai servizi religiosi sarebbe probabilmente un indicatore più idoneo, per quanto sempre problematico (Cholvy 1994; Fox 1982). 
scelta razionale può combinarsi con successo alla ricerca storica comparata e fornire una comprensione dei fenomeni politici superiore a quella degli approcci esistenti.

I partiti cristiano-democratici sono stati, e tuttora sono, una componente politica fondamentale in Europa (e, in certa misu$\mathrm{ra}$, in America Latina) ${ }^{2}$. La semplice esistenza di questi partiti, che stanno all'intersezione tra la religione e la politica ma che si sviluppano in società liberali e secolari, solleva importanti questioni teoriche sulla relazione tra religione e democrazia. Per capire cosa sono i partiti cristiano-democratici e per stabilire quale sia la relazione tra religione e politica in Europa abbiamo bisogno di una teoria della democrazia cristiana. Una teoria che dovrebbe incentrarsi sull'azione politica piuttosto che sulla demografia e l'ideologia. Invece di far riferimento alla dottrina sociale cattolica o alla proporzione di cattolici in un paese al fine di «spiegare» la presenza di quei partiti, questa teoria deve a) specificare le condizioni in cui i partiti che fanno appello agli elettori sulla base di motivi religiosi si formano e si affermano; $b$ ) tenere conto del fallimento di questi partiti ad emergere in ambienti apparentemente favorevoli; e c) determinare l'impatto di questi partiti sulla politica delle società nelle quali essi agiscono e sui rapporti tra politica e religione. In questo articolo mi concentrerò sul primo aspetto di questa teoria, offrendo un'interpretazione della nascita dei partiti confessionali che va al di là dei tradizionali riferimenti alle encicliche papali e alla cultura cattolica. Questo punto necessita però un chiarimento: spesso si assume che i partiti cristiano-democratici siano un fenomeno precipuo del dopoguerra, per nulla correlato ai partiti confessionali che li precedettero (Broughton 1988, 222; Lyon 1967, 69). È tuttavia impensabile separare i partiti confessionali anteguerra da quelli cristiano-democratici contemporanei, così come sarebbe impensabile ignorare la socialdemocrazia prima della guerra nello studio dei partiti socialdemocratici. Esiste infatti una notevole continuità tra i partiti cattolici anteguerra e quelli cristiano-democratici del dopoguerra in termini di organizzazione, di personale politico, di ideologia ed anche di strategia ${ }^{3}$. Ol-

2 Dal 1946 al 1983 i governi basati, in parte o del tutto, su partiti cristiano-democratici hanno avuto una durata variabile tra i 256 mesi in Germania e i 292 in Austria, 397 in Belgio, 456 in Olanda e in Italia (von Beyme 1985, 333). Il gruppo cristiano-democratico (Partito popolare europeo) al Parlamento europeo è il secondo in ordine di grandezza.

3 Sulle motivazioni forti del collegamento tra partiti cristiano-democratici del do- 
tretutto, il termine stesso di democrazia cristiana si ricollega al cattolicesimo antiliberale del XIX secolo. Una teoria della democrazia cristiana presuppone allora una comprensione teorica delle sue origini ${ }^{4}$.

L'articolo è diviso in quattro sezioni. Nella prima argomento la mia affermazione sulla natura molto problematica dell'attuale spiegazione delle origini dei partiti cristiano-democratici. Nella seconda delineo un modello della formazione dei partiti. Nella terza sezione impiego questo modello per spiegare la formazione dei partiti confessionali. Nella quarta confermo la validità del modello esaminando sinteticamente il caso della Francia, dove non è emerso alcun partito confessionale nonostante le circostanze favorevoli. Da ultimo, nelle conclusioni, riassumo le implicazioni del processo di formazione dei partiti confessionali sullo sviluppo politico. Utilizzo dati di sei paesi: Belgio, Olanda, Austria, Germania, Italia e Francia.

\section{La letteratura sulla democrazia cristiana}

La letteratura generale sui partiti cristiano-democratici contiene due teorie differenti che spiegano la nascita dei partiti confessionali nell'Europa del XIX secolo. La prima teoria, radicata nella sociologia storica, considera questi partiti creazioni della Chiesa cattolica di fronte allo sviluppo congiunto dell'anticlericalismo e della politica di massa (la classica asserzione di Lipset e Rokkan 1967). Una conseguenza di questa teoria è una visione ampiamente condivisa di quei partiti come parte della cultura cattolica e del contesto istituzionale. Una seconda teoria, propria della scienza politica, enfatizza il ruolo dell'élite politica conservatrice nell'appropriarsi della religione e della dottrina sociale cattolica per costruire partiti di massa e resistere alla crescita dei partiti socialisti. Questo approccio pone in secondo piano la religione a favore degli aspetti conservatori dei partiti confessionali (Lane e Ersson 1991, 108; Broughton 1988,

poguerra e partiti confessionali di prima della guerra si veda Mayeur (1980), ed anche Kalyvas (1996), in particolare il cap. 5. Per l'evidenza di una continuità tra la socialdemocrazia prima e dopo la seconda guerra mondiale si veda Przeworski e Sprague (1986).

4 Ai fini di una semplificazione utilizzo i termini democrazia cristiana, partito cristiano-democratico e partito confessionale in un'accezione generale e interscambiabile. Il termine «Chiesa», nel contesto di questo articolo, fa riferimento alla Chiesa cattolica. 
195; Layton-Henry 1982, 17; Mayeur 1980, 236). Duverger, ad esempio, sostiene che i partiti cattolici di Belgio, Olanda e Austria sono «puramente e semplicemente partiti conservatori che hanno cambiato nome» $(1966,412-413)$. Tale posizione è comunque persuasivamente criticata dalle recenti ricerche sociologiche che mostrano come i partiti cristiano-democratici influenzano il loro ambiente politico e sociale in un modo molto differente dai partiti conservatori.

Questo articolo offre una spiegazione della nascita dei partiti confessionali che va contro entrambe le teorie. La sua tesi centrale è che la formazione dei partiti confessionali è stato un esito contingente di decisioni strategiche compiute dagli attori politici. I partiti confessionali si sono costituiti a dispetto e non a causa, come di solito si assume, delle decisioni e delle azioni della Chiesa. Essi sono stati un sottoprodotto non progettato e non voluto di scelte strategiche operate dalla Chiesa in stato di costrizione. Né la stessa formazione dei partiti confessionali rientrava nelle intenzioni delle élites politiche conservatrici. Ambedue questi attori che dettero vita al processo che poi determinò la nascita dei partiti confessionali avevano, infatti, un set compatibile di preferenze che escludeva la creazione di quei partiti. Il risultato, ad ogni modo, sorprese sia la Chiesa che i leader politici conservatori. Un risultato che, consolidatosi, strutturò l'organizzazione della politica e le scelte future in modi non previsti.

Come mai allora la letteratura esistente non è riuscita ad individuare gli aspetti cruciali della formazione dei partiti confessionali? Per cominciare, si tratta di una letteratura sottosviluppata (si vedano in particolare Durand 1995; Hanley 1994; Lönne 1986; Mayeur 1980; Irving 1979; Vecchio 1979a; Fogarty 1957). In effetti è concordemente ritenuto che il fenomeno della democrazia cristiana sia stato trascurato, nonostante la sua rilevanza politica e, soprattutto, in confronto al lavoro svolto sui partiti socialisti (Hanley 1994, 1; Anderson 1991, 707; Delbreil 1990, 7; Vecchio 1979b, 53; Irving 1979, 7). Inoltre, questa letteratura soffre principalmente di cinque difetti: il funzionalismo, l'essenzialismo, il dare per scontato il problema dell'azione collettiva, l'«esogenizzazione» dei concetti di mobilitazione e di organizzazione, la carenza di una prospettiva comparata. Di seguito passerò brevemente in rassegna questi punti deboli, che non sono certo confinati solo a questo campo di ricerca.

Innanzitutto, la letteratura sui partiti confessionali è funzio- 
nalista. Le cause sono giustificate dagli esiti e l'origine storica dei partiti confessionali è derivata da un'interpretazione della loro pretesa utilità. Questa prospettiva strumentale e teleologica considera i partiti confessionali come risposte ad una varietà di stimoli, dall'industrializzazione al liberalismo, compresi la secolarizzazione, il socialismo e la modernità. Ad esempio, Lorwin afferma che «con l'industrializzazione, l'urbanizzazione e la mobilità geografica [...] le Chiese cominciarono a preoccuparsi della fede delle masse. Se l'isolamento non poteva più proteggere $i$ fedeli dalle influenze corrosive per la loro fede, speciali istituzioni dovevano incapsularli» $(1971,149)$. In modo analogo, Maier sostiene che «in una società dove le libertà della Chiesa non erano più una cosa naturale, bensì dipendevano dall'attività dei suoi membri (soprattutto laici), il cattolicesimo dovette sviluppare forme politiche. Il cattolicesimo politico è pertanto il figlio naturale del cattolicesimo per sé» $(1969,17)$ (corsivo mio; per simili considerazioni si vedano La Palombara e Weiner 1990, 28; Irving 1979, 1; von Beyme 1985, 81; De Rosa in Malgeri 1969, XIII; Lyon 1967, 69; Rommen 1950, 608). L'elemento teleologico inerente a questo approccio è stato giustamente criticato da Blackbourn (1991, 786).

Secondariamente, questa letteratura è essenzialista: la fede religiosa è vista come un sentimento collettivo naturalmente forte e l'organizzazione della politica su basi confessionali come naturale e inevitabile. L'identità religiosa esiste sempre in forma cristallizzata ed emerge nel regno della politica, di solito attraverso una veemente eruzione. Come sostiene Maier, «il modo più semplice di capire l'unità cristiano-democratica è, comunque, di capirla dal punto di vista ideologico: come una comunità di persone che ha intrapreso la politica in virtù di certe precedenti convinzioni pre-politiche» $(1969,13)$. Ad ogni modo la fede religiosa non costituisce necessariamente un'identità primordiale, ed ancor meno il fondamento di un cleavage politicizzato. Se lo costituisse, i cleavages religiosi (e i partiti religiosi) esisterebbero ovunque. Come ha sottolineato Émile Poulat $(1977,198)$, un importante studioso del cattolicesimo, l'educazione religiosa inculca un babitus che crea delle predisposizioni sociali ma che non condiziona meccanicamente il comportamento. Per parafrasare Przeworski e Sprague (1986, 54 e 182), la solidarietà tra i cattolici non è una conseguenza meccanica della loro affinità e del loro comune credo religioso. La presenza di un'ampia popolazione cattolica non garantisce l'emergere 
di un'identità politica cattolica, ossia di un'identità che incapsuli tutti i cattolici in una politica partigiana, espressa dal cattolicesimo politico e dalla formazione di un partito confessionale. Dunque, la religione non necessariamente struttura la politica, neanche in società divise dal punto di vista religioso (Laitin 1986), e l'evidenza storica indica che, nei paesi dove si formarono partiti confessionali, la religione non costituì la base di un'azione di parte prima che emergesse un cleavage religioso. L'organizzazione politica di individui sulla base della loro fede religiosa non fu né ovvia né inevitabile, poiché, come ha sottolineato Bourdieu, «il senso di un mondo sociale non si impone in modo univoco e universale» $(1987,11)$. Non fu ovvia, come ha notato Dansette: «se l'idea di un partito cattolico preoccupava il clero, essa sembrava ancor più straordinaria ai laici» (1961, 238). E non fu inevitabile, come illustrato dal caso della Francia: nonostante l'esistenza di condizioni strutturali «idonee», non nacque qui alcun partito confessionale.

La spiegazione della formazione di un partito confessionale dovrà dunque tener conto della costruzione di un'identità politica cattolica. In altre parole, essa deve endogenizzare l'identità politica e specificare come i cattolici conseguono l'equivalente di ciò che Katznelson $(1986,17-19)$ ha indicato quali terzo e quarto livello della formazione di classe. Analogamente a quanto dimostrato da Przeworski e Sprague (1986) a proposito dei partiti socialdemocratici, è l'azione dei vari attori politici (nonché l'interazione delle loro scelte strategiche) che forma l'idea sociale che i legami sono politicamente rilevanti. Nei termini usati dai primi socialisti, ciò che conta sono i liens de volonté (legami di volontà) piuttosto che i liens de nécessité (legami di necessità) (Pizzorno 1970, 46). Questa azione conduce alla politicizzazione di certi cleavages, in determinato modo e non in altro, e alla concomitante politicizzazione di certe identità. Dunque è la formazione e l'azione del movimento cattolico che creò un'identità politica cattolica piuttosto che viceversa. Una volta formatesi, le organizzazioni cattoliche alimentarono (e trasformarono) questa identità rafforzando la disciplina collettiva in modo da superare le divisioni etniche o di classe tra i cattolici.

In terzo luogo, la letteratura di sintesi dà per scontato il problema dell'azione collettiva, accettando come dato di fatto un salto diretto e automatico dagli interessi comuni all'organizzazione e all'azione politica. Ad esempio, Windell afferma che «in Prussia ci fu una combinazione di circostanze che rese utile 
la nascita di un partito cattolico. Il regno degli Hohenzollern era essenzialmente protestante; pertanto i cattolici, in quanto minoranza religiosa più forte, ebbero un interesse particolare a difendersi» (1954, 11). Noi sappiamo, peraltro (Olson 1971), che un interesse comune spesso non è sufficiente affinché emerga un'azione collettiva.

In quarto luogo, la letteratura assume che la Chiesa (o, a seconda dell'approccio, le élites politiche conservatrici) avesse sia l'incentivo che l'interesse a mobilitare le masse sulla base di un richiamo religioso e ad organizzarle, in organizzazioni di massa permanenti prima e in partiti confessionali poi. Dunque, la creazione di organizzazioni laiche di massa da parte della Chiesa è generalmente considerata una scelta priva di costi e dotata solo di ricavi positivi (McLeod 1986). Con ciò, questa letteratura riecheggia la diffusa tendenza degli studi classici sulla mobilitazione politica e sulla creazione delle organizzazioni a focalizzarsi esclusivamente sul termine ultimo: le masse da organizzare. Le decisioni e le scelte degli attori vengono abitualmente ignorate, mentre la mobilitazione e l'organizzazione sono date per scontate. Si ritiene che gli imprenditori politici abbiano sempre un interesse a mobilitare ed organizzare le persone e che sempre intraprendano questo tipo di azioni. Tuttavia, come sottolineano Rosenstone e Hansen, questa assunzione è sbagliata: «la mobilitazione non è un'evenienza universale o costante. I leader politici non provano a mobilitare chiunque, né lo fanno continuamente. La mobilitazione, dopo tutto, non è il loro vero obbiettivo [...]. La mobilitazione è una strategia che essi possono seguire, ma non è né la sola né quella sempre migliore» $(1993,30)$. Pertanto, la mobilitazione, al pari della formazione dei partiti politici e delle organizzazioni di massa, deve essere endogenizzata nel processo di formazione dei partiti.

Da ultimo, mentre i pochi studi comparati tendono ad essere mere giustapposizioni di casi, la maggior parte dei lavori è costituita da studi del caso e i loro autori affermano di solito l'unicità dell'oggetto investigato. Ad esempio, la frase iniziale del libro di Bakvis è che «il partito cattolico olandese è unico» (1981, 1; per affermazioni dello stesso tenore si vedano Carton de Wiatr in Guyot de Mishaegen 1946, I sul Belgio; e Mann 1968, 212 sulla Germania). Oltre a costituire un impedimento alla ricerca, la mancanza di una prospettiva comparata è la fonte principale degli elementi di debolezza. Così, ad esempio, Molony pone l'accento sulle implicazioni causali della guerra: 
«il fattore storico che contribuì più di ogni altro alla formazione del PPI fu senza dubbio la prima guerra mondiale. Dopo un conflitto nel quale i cattolici avevano combattuto ed erano morti per il loro paese, era impossibile negar loro un ruolo in tempo di pace» $(1977,47)$. Questa affermazione perde di fondamento allorché si guarda alla Francia, dove un processo simile ha prodotto esattamente l'esito opposto: mentre da un lato la guerra alimentò la fratellanza tra i francesi e contribuì al processo di integrazione del cattolicesimo nella Repubblica, dall'altro essa allontanò i cattolici francesi dalla politica spingendoli alla religiosità individuale (Bosworth 1962, 31).

Diversamente da questa letteratura, la spiegazione che segue endogenizza l'identità politica, la mobilitazione e l'organizzazione nel processo di formazione partitica. Nel far ciò, questo modello fornisce alcuni microfondamenti della teoria di Lipset e Rokkan e delinea la sua applicabilità. Il processo di formazione dei partiti è «spacchettato» mediante l'impiego di un approccio analitico che è come una «spiegazione attraverso meccanismi» (Elster 1989, 1). Vengono quindi qui specificati un semplice modello di formazione partitica che include le preferenze dei due attori principali e che ricostruisce la mappa delle loro opzioni, nonché le previsioni che da tale modello derivano. Nella sezione seguente fornirò una descrizione astratta del processo, mentre nella terza approfondirò gli elementi di dettaglio.

\section{Un modello di formazione partitica ${ }^{5}$}

Il processo di formazione dei partiti ha luogo in tre ampi periodi di tempo. Ha inizio con la prima offensiva dei liberali ai privilegi della Chiesa ( $\mathrm{t} 1$ ) e si conclude con la formazione dei partiti confessionali ( $\mathrm{t} 3$ ). Durante il periodo $\mathrm{t} 1$ sono presenti tre attori: la Chiesa, i politici conservatori e i politici liberali. Qui ripongo la mia attenzione in primo luogo sulle preferenze e sulle strategie della Chiesa e dei conservatori ${ }^{6}$. La figura 1 illustra la sequenza delle decisioni.

5 Sebbene questo modello sia qui utilizzato in riferimento all'Europa del XIX secolo, esso è potenzialmente applicabile a tutti i paesi nei quali ha luogo un processo di formazione del sistema partitico.

6 Considero che gli esponenti politici liberali abbiano preferenze simili a quelle dei conservatori (la rielezione). Essi sono pertanto esogeni rispetto al modello sia per 

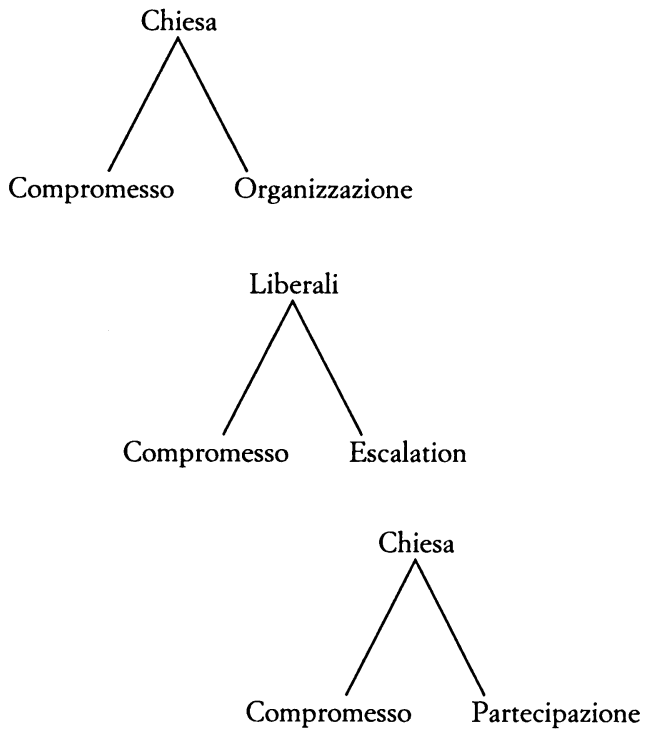

t2

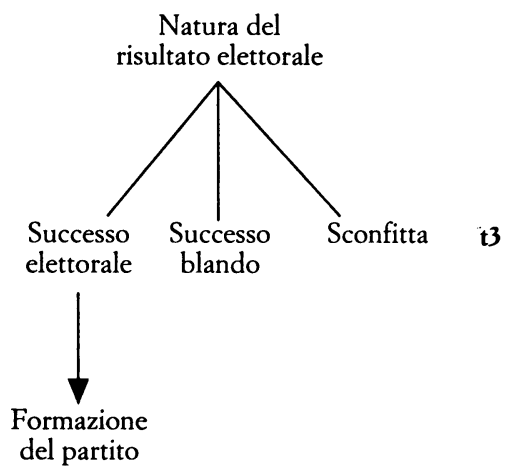

FIG. 1. Il processo di formazione dei partiti confessionali (Belgio, Olanda, Austria, Germania, Italia).

questione di semplicità, sia perché la loro strategia resta costante lungo tutto il processo (offensiva anticlericale). Ai fini di questo studio tratto la Chiesa come attore unitario, intendendo per essa sia la gerarchia (o episcopato) sia la curia romana (o Vaticano). Questa è un'assunzione ragionevole in virtù della struttura piramidale della Chiesa, dell'estremo grado di centralizzazione e di gerarchizzazione, e della sua natura transnazionale (Vaillancourt 1980,12). Ovviamente, questa assunzione ha una sua validità solo nel contesto di questo studio e in riferimento alle decisioni fondamentali, quale la reazione alle offensive anticlericali. 
Primo periodo (t1). La Chiesa è un attore autointeressato che massimizza il potere, generalmente concepito come capacità di forgiare o influenzare la società (Gramsci, citato in Fulton 1987, 212 e Weber 1963, 235-236; Chapman 1962, 153). Un significativo indicatore del potere è l'ammontare dei diritti e dei privilegi di cui la Chiesa beneficia, come la fornitura esclusiva o preferenziale di certi servizi - in particolare l'istruzione. I partiti liberali, al potere in tutti i sei paesi in esame, cominciarono ad approvare, o manifestarono l'intenzione di farlo, una prima serie di leggi, soprattutto in materia di istruzione e di famiglia, che ridussero in modo decisivo il potere della Chiesa («prime minacce»). Allo stesso tempo, essi bloccarono l'accesso prima diretto e privilegiato della Chiesa ai centri decisionali.

Nel periodo t1, la Chiesa può scegliere tra due strategie: a) giungere ad un compromesso e quindi accettare la maggior parte delle misure anticlericali («non combattere» o NF); e $b$ ) reagire a quelle offensive, da sola e al di fuori del processo politico, essenzialmente attraverso la creazione di organizzazioni di massa («strategia organizzativa» o $O)^{7}$. Nella ricerca dei mezzi per un'azione politica orientata a preservare la sua influenza dall'offensiva dei liberali, la Chiesa sottostà ad un fondamentale trade-off: cercare di massimizzare la sua influenza (i) senza, peraltro, patire inaccettabili costi organizzativi $(c)$. Mentre la perdita di influenza della Chiesa a seguito delle offensive anticlericali è ovvia, il problema dei costi organizzativi richiede ulteriori precisazioni.

I costi organizzativi sono per lo più, anche se non esclusivamente, connessi al controllo. Per la Chiesa cattolica il controllo è una questione centrale (Rahner et al. 1968). La Chiesa è un'organizzazione fermamente gerarchica, rigidamente stratificata e altamente centralizzata con una struttura piramidale - e queste caratteristiche furono ancor più rafforzate durante la seconda metà del XIX secolo (Blackbourn 1991, 780). Al vertice si trova il Va-

7 Se l'esatto contenuto di queste strategie, nonché la loro forma di espressione, varia tra paese e paese, i loro elementi fondamentali sono simili. La creazione di organizzazioni di massa è, al tempo $t 1$, il solo modo possibile di opporsi, dal momento che $\mathrm{i}$ collegamenti della Chiesa ai centri decisionali sono stati tagliati. Si può pensare che la formazione di organizzazioni di massa sia un segnale che la Chiesa manda ai liberali. La formazione di un partito non ha molto senso in questo momento, poiché non ci sono ancora organizzazioni di massa. Sia la partecipazione al processo elettorale che l'organizzazione di un nuovo partito avrebbero richiesto un'organizzazione di massa che non esisteva al tempo $t 1$. 
ticano e appena al di sotto stanno gli episcopati delle Chiese nazionali. Il basso clero è sottoposto ad una stretta sorveglianza da parte della gerarchia: il compito del prete è semplicemente quello di aiutare il suo vescovo «nell'esercizio delle sue funzioni pastorali» (Dondeyne 1964, 46). I cattolici laici, membri della Chiesa, erano esclusi da ogni sorta di potere di governo all'interno della Chiesa e stavano al livello più basso della Chiesa stessa, al punto da essere definiti canonicamente in termini negativi come «coloro che non partecipano al potere di giurisdizione $\mathrm{e}$ specialmente di ordine [nella Chiesa]» (Vermeersch e Creusen, citato in Congar e Varillon 1947, 8; Gambasin 1969, 14). Come papa Pio X ricordò ai cattolici nella sua enciclica del 1906 Vebementer Nos, «la Chiesa è per sua natura una società ineguale; è costituita di due categorie di persone: i pastori e il gregge. Solo la gerarchia promuove e controlla. [...] Il dovere della massa è di accettare di essere governata e di seguire con sottomissione gli ordini di coloro che la guidano» (citato in Aubert 1975, 155). L'arcivescovo di Rouen è ancora più esplicito: «i laici non svolgono alcuna funzione negli affari della Chiesa» (citato in Dansette 1961, 236). E ovvio che la Chiesa possa meglio controllare $i$ suoi membri allorché abbia l'esclusiva della loro fedeltà e goda di un monopolio istituzionale della loro rappresentanza.

In questo quadro, il dilemma della Chiesa al tempo t1 è il seguente: giungere ad un compromesso le consente di mantenere il controllo sui suoi membri e di non inimicarsi i liberali, ma la sua influenza complessiva diminuisce. Opporsi produce il risultato contrario. La creazione di organizzazioni di massa ad opera di un'organizzazione già esistente, gerarchica, centralizzata, autoritaria e rigidamente stratificata, qual è la Chiesa, genera esternalità negative per essa, soprattutto perché riduce il grado di controllo esercitato sui suoi membri, i cattolici laici ${ }^{8}$. L'organizzazione laica di massa richiede più del solo reclutamento attivo dei membri. Richiede la formazione di una struttura orga-

8 Recenti studi sui costi di transazione e sulla teoria aziendale sottolineano l'importanza del controllo di un'azienda nella sua relazione con gli agenti. Anche se questi lavori si occupano principalmente dei profitti monetari piuttosto che dei policy benefits e delle fusioni piuttosto che delle divisioni, e nonostante sia difficile assimilare la posizione dei cattolici laici a quelle presenti nelle aziende, il seguente punto è comunque valido: «in un mondo di costi di transazione e di contratti incompleti, i diritti residuali di controllo ex post saranno importanti, perché, attraverso la loro influenza sull'utilizzo dei beni, condizioneranno il potere di negoziazione ex post e la divisione del surplus ex post in una relazione» (Hart 1989, 1766). 
nizzativa e di una logica d'azione totalmente nuove, nonché la contemporanea formazione di gerarchie parallele potenzialmente competitive. A sua volta, ciò produce nuove élites, nuove identità collettive ed una competizione interorganizzativa; può sovvertire la relazione asimmetrica tra laici e clero, e minare la struttura gerarchica della Chiesa. Oltre a tutto ciò, la creazione di organizzazioni di massa è di solito un processo costoso, terribilmente lento, arduo e tedioso, scelto solo quando non sono disponibili altre opzioni (Luebbert 1991).

La Chiesa preferirà, dunque, la creazione di organizzazioni di massa al compromesso solo nel momento in cui la conservazione della sua influenza eccede i costi organizzativi. Ciò è vero quando $a$ ) l'offensiva anticlericale è, o si attende che sia, dura; e b) non vi sono altre modalità d'azione: gli alleati conservatori sono politicamente deboli, il regime solido e i mezzi di azione extraparlamentare (quale la possibilità di un colpo militare) non disponibili. A queste condizioni la Chiesa accetterà alcune misure di perdita di controllo e combatterà creando organizzazioni di massa.

Nel periodo t1 la Chiesa ha un orizzonte temporale limitato: vede terminare il processo al tempo $t 2$ e non prevede né la partecipazione diretta alle elezioni, né gli effetti che da ciò possono conseguire. Questo si verifica perché la creazione di organizzazioni di massa fu adottata come strategia difensiva lenta e di lungo periodo, che avrebbe protetto la Chiesa e i suoi membri da ulteriori offensive dei liberali. Il proteggersi, conseguito attraverso l'isolamento dei cattolici dallo «stato liberale» e la creazione di una subcultura distinta, avrebbe tutt'al più potuto condurre alla riconquista dello stato a partire dal basso (per maggiori dettagli si veda la terza sezione). La Chiesa non era sola, peraltro, a credere che la democrazia liberale non si sarebbe conservata a lungo: $i$ primi socialisti avevano aspettative e progetti simili (Przeworski e Sprague 1986). Insomma, per quanto combinata con un appoggio non sistematico agli esponenti politici conservatori, questa era una strategia non parlamentare in virtù della quale la Chiesa avrebbe combattuto da sola e al di fuori dell'arena elettorale. A tale scelta fu data una giustificazione ideologica di aperto rigetto verso la democrazia liberale, come espresso al meglio dall'enciclica papale Syllabus pubblicata nel 1864. Il rigetto della politica elettorale raggiunse il punto estremo in Italia, dove il papa proibì formalmente ai cattolici di votare. 
Secondo periodo ( $t 2$ ). Nel periodo t2 i liberali intensificarono la loro offensiva contro la Chiesa. Lo fecero sia per motivazioni intrapartitiche (pressioni delle loro correnti radicali), che per considerazioni strategiche (l'anticlericalismo fu visto come una delle poche armi effettive del liberalismo borghese contro il socialismo nella lotta per i voti della classe operaia). La creazione di organizzazioni cattoliche di massa, infatti, fornì ai liberali un buon motivo per quella escalation (il «mantenimento dell'integrità dello stato contro $\mathrm{i}$ clericali»), anche se all'inizio non li minacciava politicamente, dato che le organizzazioni cattoliche erano tenute fuori dall'arena elettorale. $\mathrm{Al}$ tempo $\mathrm{t} 2$, dunque, la Chiesa ha di fronte a sé un pericolo più grave ( $i$ diminuisce) ed è costretta a fare nuove scelte. Essa può: $a$ ) non muoversi dalla sua strategia organizzativa iniziale e restare fuori dal processo elettorale $(O)$; oppure $b$ ) fare ingresso direttamente nel processo politico organizzando e appoggiando pienamente coalizioni elettorali che agiscano come suoi subappaltatori politici provvisori («strategia partecipativa» o $P$ ); oppure ancora $c$ ) creare un partito confessionale $(C P)$.

La differenza fondamentale tra le strategie organizzativa e partecipativa è il loro scopo. La strategia partecipativa consiste nel combattere contro l'anticlericalismo al fianco di alleati e all'interno del processo elettorale. Essa rappresenta una rottura con la prassi precedente di un appoggio non sistematico nei confronti di singoli cattolici indipendenti o di distinte fazioni di conservatori. A questo punto la Chiesa cerca di specificare una piattaforma (l'equivalente di un contratto) come base per la formazione di un'ampia coalizione di forze politiche conservatrici. Il rapporto tra la Chiesa e i suoi alleati assume infatti la forma di una mutua relazione di subappalto, sulla base della quale gli alleati politici conducono una lotta politica per la difesa della Chiesa in nome del cattolicesimo (sebbene non come rappresentanti ufficiali della Chiesa) e in cambio la Chiesa offre loro sostegno elettorale mobilitando i suoi membri, in modo particolare mediante le sue nuove organizzazioni di massa.

L'aperta partecipazione della Chiesa al processo politico mediante la costituzione di coalizioni filo-ecclesiastiche sostenute elettoralmente dalle organizzazioni cattoliche di massa produce ovvi benefici: il mantenimento dell'influenza della Chiesa attraverso una sfida aperta e diretta nei confronti dei liberali. La strategia partecipativa aggrava, peraltro, i deleteri effetti dell'organizzazione di massa e indebolisce ulteriormente il legame 
tra la Chiesa e i suoi membri. Ciò accade perché il controllo può essere mantenuto più facilmente quando le organizzazioni cattoliche hanno un raggio d'azione ristretto. Il grado di controllo che la Chiesa esercita sui suoi membri attivamente politicizzati diminuisce in modo sostanziale allorché la fonte del potere e della legittimità degli attivisti cattolici passa dalla Chiesa agli elettori. La partecipazione in un sistema politico democratico spalanca le porte ad un'arena interamente nuova, fuori e al di là del regno della Chiesa, sia a livello individuale (per i membri delle organizzazioni cattoliche), sia a livello collettivo (per le organizzazioni cattoliche).

La partecipazione politica in un regime parlamentare rappresentativo crea, dunque, «i mezzi per un attivismo del clero e dei laici sottratto alla supervisione diretta dell'episcopato» (Gellott 1987, 68). Ciò produce tre effetti. Primo, in questa arena sono disponibili importanti payoffs non connessi alla Chiesa; il successo personale e collettivo è adesso il risultato più della partecipazione attiva e dell'azione autonoma, e meno dell'obbedienza passiva e della deferenza alla gerarchia. Secondo, la partecipazione alla politica democratica introduce i valori democratici secolari dell'autonomia individuale e dell'eguaglianza nel mondo cattolico della gerarchia e dell'obbedienza di origini divine. Terzo, la partecipazione porta gli attivisti cattolici a spingere per una maggiore autonomia d'azione e per un'ulteriore partecipazione al processo politico dal momento che questo dà loro la sensazione, per la prima volta, che la politica possa far progredire la loro causa. Inoltre, la partecipazione al processo elettorale produce una perdita di vantaggio organizzativo per la Chiesa. Utilizzando un concetto tratto dall'economia politica, possiamo pensare alla Chiesa come ad un «interesse concentrato» alla ricerca di rendite parassitarie (rent-seeking). Il vantaggio di godere di questo tipo di interessi in confronto agli interessi diffusi è la capacità di sopraffare il fenomeno del freeriding. Tuttavia, il vantaggio viene meno quando i partiti si impadroniscono dei problemi, che diventano così un elemento della politica di parte. Verdier $(1994,17-20)$ ha dimostrato che organizzare un'ampia coalizione e politicizzare una determinata questione incrementa $i$ costi della ricerca di rendite parassitarie 9 . Infine, l'attività politica visibile da parte della Chiesa com-

9 Questo accade per tre ragioni: primo, rendere di parte un problema richiede «spese di campo», vale a dire spese di mobilitazione e di organizzazione; secondo, il set 
porta il pericolo di rappresaglie ulteriori e dirette delle forze anticlericali. Come ebbe a sottolineare il vescovo della città francese di Valence, «se i cattolici esigessero dai candidati di favorire la loro Chiesa, non potrebbero lamentarsi se altri esigessero dai candidati di esserle contro» (citato in McManners 1972, 174).

Spesso si suppone erroneamente che la creazione di un partito confessionale, vale a dire di un partito associato in modo permanente ed esclusivo alla causa religiosa e organizzativamente connesso alla Chiesa, rappresenti la protezione politica ideale per la Chiesa. In effetti, la formazione di un tale partito aggrava ancor più gli effetti deleteri dell'organizzazione e della partecipazione e riduce il controllo. Adesso sono gli elettori, più che la Chiesa, a diventare la fonte stabile di potere e di legittimazione per gli attivisti cattolici. L'attivista di un partito cattolico finisce per essere meno controllato dalla Chiesa e meno legato ad essa rispetto a quando era un semplice membro della massa dei fedeli o del basso clero. Allorché il leader del Zentrum cattolico tedesco, Ludwig Windthorst, si rifiutò di obbedire agli ordini del papa e di votare per il bilancio militare di Bismark, rese assolutamente chiaro un punto: «il Zentrum - affermò in un famoso discorso - vive solo e soltanto della fiducia della gente; esso non sottostà agli ordini di nessun altro e gli è [...] pertanto richiesto, più che ad ogni altro partito, di tener conto del polso della gente» (citato in Anderson 1986, 112). La creazione di un partito confessionale, in più, sovverte le tradizionali relazioni all'interno della Chiesa. In quanto parlamentare, e non come semplice sacerdote o laico, un cattolico instaura un differente rapporto con un vescovo. Quando il sacerdote e deputato del Zentrum Theodor Wacker asserì nel 1914 che la gerarchia non aveva voce in capitolo negli affari del partito, egli parlava, come rilevato da Ross, «da politico effettivo, non da prete o da teologo» $(1976,62)$.

Infine, ma non certo di minore importanza, la creazione di un partito confessionale mina in modo decisivo la pretesa universalistica della Chiesa e genera nuovi rischi per il modo in cui la Chiesa e la religione si associano a cause politiche impopola-

delle alternative possibili di policy diminuisce quando un problema specifico deve essere trasformato in un problema generale in modo da attrarre un sostegno pubblico; e, terzo, il rischio che gli agenti politici si sottraggano alle loro responsabilità diventa più grande. 
ri. Il richiamo universalistico è sempre stato una questione primaria per la Chiesa, tanto che la tesi per cui «la Chiesa non può diventare un partito perché è di tutti» fu inserita nell'enciclica papale Sapientiae Christianae del 1890.

D'altro canto, se la creazione di un partito confessionale diminuì il controllo della Chiesa, non per questo aumentò la sua influenza. Insufficiente per la trasformazione della Chiesa in partito, un partito confessionale laico tende a non essere controllato facilmente a causa dei problemi agente-principale connessi ai fattori sopra menzionati. $\mathrm{Ne}$ consegue che un partito come questo non sarà necessariamente un agente politico della Chiesa migliore del partito conservatore, che agisce come subappaltatore politico della Chiesa e dipende dalle sue organizzazioni di massa. Infatti, sin dal momento in cui le organizzazioni cattoliche di massa vengono incorporate in un partito confessionale e poste sotto la direzione dei leader laici più che della Chiesa, quel partito rivela di essere un agente della Chiesa peggiore delle élites politiche conservatrici ${ }^{10}$. Ad ogni modo, la formazione di partiti confessionali non è una buona scelta per la Chiesa anche assumendo che tali partiti saranno agenti della Chiesa migliori di quelli conservatori: il costo della formazione di un partito è ancora troppo elevato per essere compensato da un modesto aumento d'influenza (la figura 2 illustra questo punto). Ne consegue che, sebbene sia comunque un'opzione costosa, l'uso di politici conservatori quali subappaltatori politici controllati dall'organizzazione ecclesiastica è preferibile alla formazione di un partito che faccia della religione la sua missione fondamentale e minacci di diventare un concorrente per la rappresentanza dei membri della Chiesa. Questo spiega perché, come affermato da Mayeur, «tra la creazione di organizzazioni del tipo dei gruppi d'interesse e quella di partiti politici la Chiesa preferì chiaramente la prima soluzione» $(1980,8)$.

Data la rapida diminuzione d'influenza, al tempo t2 la Chie-

10 La minaccia della Chiesa di ritirare l'appoggio ai conservatori poteva apparire non credibile alla luce dell'alternativa liberale anticlericale. Tuttavia, dal momento che i partiti conservatori erano fragili alleanze di notabili, la Chiesa poteva manovrare a proprio vantaggio le varie correnti mettendole l'una contro l'altra. Inoltre, essa poteva fare appello alle frazioni conservatrici interne ai partiti liberali (come fece in Italia nel 1913). Certo, la Chiesa poteva esercitare pressioni anche nei confronti dei partiti confessionali rivolgendosi direttamente ai fedeli dal pulpito, ma ciò era difficile e costoso, in considerazione delle risorse superiori delle organizzazioni cattoliche (rispetto ai partiti conservatori) e della fedeltà di un gran numero di credenti ai partiti confessionali. 


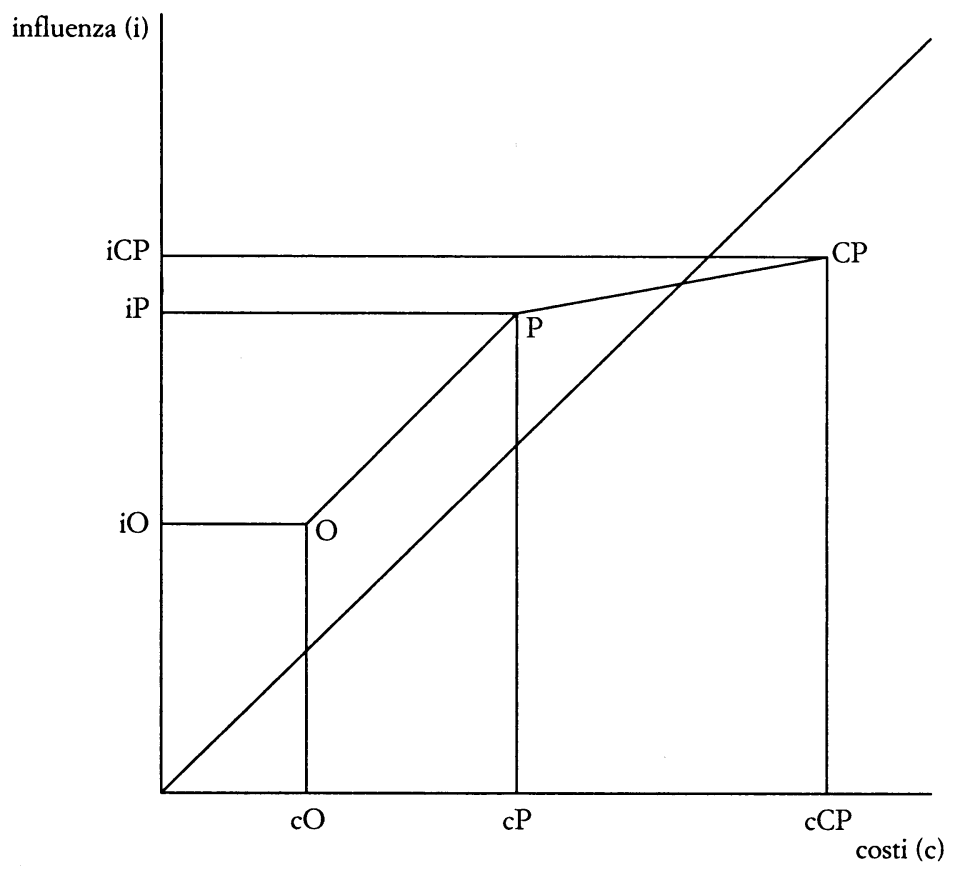
Legenda: Strategie O: strategia organizzativa
P: strategia partecipativa
$\mathrm{CP}$ : formazione di un partito confessionale
Costi cO: costi di O
cP: costi di $P$
cCP: costi di CP
Influenza $\mathrm{iO}$ : influenza generata da $\mathrm{O}$
iP: influenza generata da $\mathrm{P}$
iCP: influenza generata da $\mathrm{CP}$

FIG. 2. Costi e benefici delle strategie della Chiesa ( $\mathrm{t} 2$ ).

sa sceglie di sostenere i costi della strategia partecipativa piuttosto che restare ferma alla strategia organizzativa iniziale - soprattutto se le opzioni politiche alternative (quali quelle sopra descritte) rimangono indisponibili. La Chiesa si aspetta che le ampie coalizioni di forze conservatrici costruite a difesa della sua piattaforma e rafforzate dalle organizzazioni cattoliche di massa siano in grado di intimidire elettoralmente i liberali, fino a fermare le loro offensive anticlericali. Essa si aspetta inoltre, a 
seguito dei buoni risultati elettorali di quelle coalizioni, di negoziare con i liberali il futuro impiego elettorale delle sue organizzazioni (ad esempio, il loro ritiro dall'arena elettorale in cambio di una restaurazione dei suoi diritti) ${ }^{11}$. Insomma, $P$ è preferito sia ad $O$ sia a $C P$ : la predizione del modello è allora che la Chiesa, al tempo $t 2$, sceglie di non formare un partito confessionale.

Accanto alla Chiesa, vi è un altro attore che entra a far parte di questo processo al tempo t2: i conservatori. Essi hanno una scelta fra tre strategie: a) chiedere/accettare solo un sostegno non sistematico da parte della Chiesa $(U) ; b)$ chiedere/accettare un sostegno sistematico da parte della Chiesa, che così attuerebbe la sua strategia partecipativa $(P) ; c)$ creare un partito confessionale $(C P)$. Gli esponenti conservatori sono notabili, membri di fragili correnti parlamentari ben disposte verso la maggior parte delle rivendicazioni della Chiesa. Tuttavia, essi desiderano più di ogni altra cosa di proseguire la loro carriera politica puntando alla rielezione (Verdier 1994; Geddes 1991). Se da un lato l'organizzazione di massa può fornire loro indubbi benefici in un periodo di allargamento previsto o effettivo del suffragio elettorale, dall'altro essa comporta dei costi inerenti alla loro sopravvivenza politica. Essendo notabili tradizionali, i parlamentari conservatori non gradiscono la prospettiva di dover creare un partito di massa, poiché ciò porterebbe alla formazione di una nuova classe politica professionale che minaccerebbe appunto la loro sopravvivenza politica (Irvine 1989; Pizzorno 1970) ${ }^{12}$. Da ciò deriva che i politici conservatori preferiscono cercare altrove le risorse organizzative - una soluzione meno costosa e più sicura. Le organizzazioni di massa della Chiesa sono molto appetibili, soprattutto quando la Chiesa garantisce di tenerle fuori dall'arena politica; ciò consente ai conservatori, come il presidente del consiglio italiano Giovanni Giolitti nel 1913, di «acquistare a buon mercato» i voti cattolici (De Rosa 1972, 352). Oltretutto, poiché le issues sono necessarie alla mobilitazione (Rosenstone e Hansen 1993, 34) e poiché i politici che sono stabilmente perdenti alle elezioni (come erano a questo punto i conservatori) tentano di introdurre nuove

11 Un esempio in tal senso fu l'accordo tra il Vaticano e lo stato fascista in Italia nel 1929.

12 Ciò spiega perché la grande maggioranza dei partiti di massa fu «formata dall'esterno», da attori che operavano al di fuori del processo politico. 
dimensioni di competizione (Riker 1986), gli esponenti conservatori vengono attratti dalla religione anche in quanto issue. Tuttavia, essi rigettano la prospettiva di un'associazione stabile con la religione, poiché ciò restringe il loro spazio di manovra e la loro autonomia d'azione. La religione è un magnete elettorale e, in quanto tale, attrae e respinge (Van Kersbergen 1994, 25). Insomma, i conservatori preferiscono una situazione nella quale essi possono essere parlamentari cattolici non obbligati «né verso un partito né verso una confessione» (Anderson 1981, 139), piuttosto che cattolici parlamentari, stabilmente vincolati alla rappresentanza elettorale di interessi confessionali e alla difesa della Chiesa e della religione. Pertanto, al tempo $t 2$, i conservatori opteranno per la strategia partecipativa: $P$ è preferito sia ad $U$ sia a $C P$.

Date le preferenze degli attori, l'intensificazione delle offensive anticlericali e la non disponibilità di modi alternativi di azione, la principale previsione del modello è che al tempo t1 la Chiesa opterà per la strategia organizzativa, e che al tempo t2 sia la Chiesa che le élites politiche conservatrici opteranno per la strategia partecipativa piuttosto che per la formazione di un partito confessionale. In altre parole, $i$ due attori politici dovrebbero selezionare le strategie che escludono la formazione di partiti confessionali. La creazione di partiti confessionali è inefficiente per entrambi, dal momento che essa determina sostanzialmente costi più alti e, se mai, progressi marginali rispetto alla strategia partecipativa. Quest'ultima costituisce un equilibrio fondato su un mutuo accordo di scambio delle attività non gradite ma necessarie dei due attori: la Chiesa affida alle élites politiche conservatrici la lotta elettorale e politica contro le riforme anticlericali e le élites politiche conservatrici lasciano alla Chiesa il compito di sostenerle mediante le organizzazioni di massa.

Tuttavia, a dispetto della previsione del modello, i partiti confessionali sono sorti in cinque paesi. Abbiamo dunque bisogno di una spiegazione della formazione dei partiti confessionali date le preferenze e le scelte dei principali attori politici (vale a dire, nonostante il loro rigetto di questa prospettiva).

Il mio argomento è il seguente. I partiti confessionali (al tempo t3) sono nati perché la Chiesa cadde vittima del successo della propria strategia. La strategia partecipativa produsse importanti vittorie elettorali che consentirono agli attivisti cattolici di premere con successo per la formazione di partiti confessio- 
nali. La Chiesa non poté trasformare se stessa in un partito politico, né congelare il processo e impedire alle sue organizzazioni di massa di evolvere in partiti politici. I successi elettorali diedero agli attivisti cattolici il potere politico che essi usarono per opporsi al loro rientro nella Chiesa o alla loro ricollocazione ai margini della vita politica.

La spiegazione stilizzata che segue è fondata su due categorie di fonti: la prima riguarda vecchie monografie storiche e studi del caso che sono stati spesso trascurati dalla letteratura generale; la seconda fa riferimento a recenti studi del caso sociologici e storici che si sottraggono alla maggior parte dei difetti della letteratura di sintesi ${ }^{13}$. Questi studi rivelano, spesso in modo frammentario, un quadro del processo di formazione dei partiti più ricco, più dettagliato e in definitiva molto diverso rispetto alla letteratura di sintesi. Il tentativo della Chiesa di reprimere la formazione di questi partiti e la simile avversione dei conservatori di fronte a tale prospettiva emergono con grande chiarezza. E tuttavia, privi di una prospettiva sia teorica che comparata, questi studi non riescono a fornire una spiegazione alternativa, generale e comprensiva della formazione dei partiti confessionali.

\section{L'origine dei partiti confessionali: una spiegazione rivisitata}

Il processo di formazione partitica si differenziò su vari punti nei cinque paesi in esame e $\mathrm{i}$ singoli partiti confessionali che nacquero non si assomigliavano per molti aspetti. Tuttavia, tutti i partiti confessionali sorsero a seguito di una mobilitazione basata su un richiamo religioso, e il modello e le dinamiche di fondo del processo che condusse alla loro formazione erano fondamentalmente simili (Aubert 1982, 191). Oltretutto i casi in esame sono contigui (Sewell 1992). La formazione dei partiti confessionali ebbe luogo nello stesso periodo (1870-1920), in paesi europei che affrontavano sfide analoghe: l'industrializzazione, la democratizzazione, la crescita dei movimenti della classe operaia e forti Chiese cattoliche minacciate dai governi liberali (Luebbert 1991, 60-62). L'equivalenza analitica dei casi permette un trattamento comparato ed anche complementare (si

13 Per i riferimenti ad alcune fonti di questa spiegazione si rinvia a Kalyvas (1996). 
TAB. 1. Cronologia della formazione dei partiti confessionali

\begin{tabular}{lccccc}
\hline & Belgio & Olanda & Austria & Germania & Italia \\
\hline Prime minacce & $1850-1860$ & $1857-1878$ & 1866 & $1866-1870$ & $1850-1861$ \\
Strategia organizzativa & $1864-1878$ & $1868-1878$ & $1868-1874$ & $1867-1870$ & $1874-1913$ \\
$\begin{array}{l}\text { Offensiva anticlericale } \\
\text { Strategia partecipativa }\end{array}$ & $1878-1884$ & $1878-1888$ & $1867-1874$ & $1870-1878$ & $1861-1890$ \\
$\begin{array}{l}\text { Successo elettorale } \\
\text { Formazione }\end{array}$ & 1884 & 1888 & 1887 & $1870-1871$ & $1913-1919$ \\
$\begin{array}{l}\text { Istituzionalizzazione/ } \\
\quad \begin{array}{l}\text { centralizzazione } \\
\text { del partito }\end{array}\end{array}$ & 1884 & $1879 a, 1888 b$ & 1890 & 1871 & $1913 ; 19$ \\
\hline
\end{tabular}

Legenda

Prime minacce: prime offensive liberali contro la Chiesa.

Strategia organizzativa: la Chiesa combatte da sola ed al di fuori del sistema politico.

Offensiva anticlericale: massima intensità dell'offensiva anticlericale. elettorale.

Strategia partecipativa: la Chiesa combatte con alleati politici all'interno dell'arena

Successo elettorale: primi successi in elezioni.

Formazione: formazione ufficiale di un partito cattolico.

Istituzionalizzazione/centralizzazione del partito: creazione in tutto il paese di ramificazioni locali controllate da uffici centrali.

Note

a Formazione del partito calvinista.

$b$ Formazione del partito cattolico.

veda la tab. 1). Gli attori principali (la Chiesa, le élites politiche conservatrici, i liberali) erano sostanzialmente molto simili in tutti e sei i paesi - in termini delle loro preferenze, così come per il contesto del processo che stiamo analizzando. Ciò è a maggior ragione vero per le Chiese cattoliche nazionali, che erano parte di una più ampia istituzione sovranazionale, centralizzata e gerarchica.

Tentativi sparsi da parte di imprenditori politici di creare partiti politici fondati su un richiamo religioso prima del 1860 erano sempre falliti. Questi tentativi furono ignorati sia dalla Chiesa che dalla parte di elettorato composta di cattolici fedeli. Il cattolicesimo politico non esisteva. La maggior parte dei cattolici praticanti vedeva se stessa come conservatrice o monarchica, spesso persino come liberale. Il suo comportamento politico era coerente con queste identità. Tra i vari partiti, le fazioni conservatrici esistenti di solito si occupavano della protezione dei diritti e dei privilegi della Chiesa, ma ciò non fu sempre la loro priorità fondamentale, né d'altra parte i partiti liberali manifestarono in 
continuazione un atteggiamento anticlericale. La Chiesa agiva politicamente in modo pragmatico, trattando direttamente con $\mathrm{i}$ governi, sia conservatori che liberali, proprio come molti dei gruppi d'interesse. In Europa, durante l'epoca pre-parlamentare, la Chiesa seguì la strategia del concordato, ossia di accordi tra $i$ governi nazionali e il Vaticano che delinearono l'estensione dei diritti e dei privilegi della Chiesa. Le discrete pressioni di ministri per specifiche concessioni alla Chiesa furono preferite alla mobilitazione dei cattolici laici. La Chiesa, infatti, si oppose decisamente alla mobilitazione a suo sostegno dei suoi membri (May 1973, 82; Schorske 1967, 363). «La conclusione logica di una tale strategia fu l'acquiescenza ecclesiastica sulle questioni politiche in cambio della protezione degli interessi confessionali della Chiesa da parte dei conservatori e dei governi» (Anderson 1981, 194). In questo contesto, il peso politico dei cattolici laici fu necessariamente minimo, una situazione coerente con la dottrina e l'organizzazione della Chiesa: «i laici ebbero certamente un ruolo importante», afferma Aubert $(1982,192)$, «ma come membri dell'establishment, nella misura in cui erano parte del vertice della classe di governo».

Date le preferenze degli elettori, le preoccupazioni delle élites di partito e la strategia della Chiesa, niente faceva supporre che sarebbero sorti partiti confessionali forti e vitali. Al contrario, in tutta Europa i sistemi partitici sembravano svilupparsi lungo la consueta dicotomia conservatori-liberali. Tuttavia, a cominciare dal decennio tra il 1860 e il 1870 , i governi, che stavano operando una centralizzazione del potere, lanciarono una serie di offensive contro i diritti della Chiesa in vari paesi: in Belgio, in Olanda, in Austria, negli stati germanici (e, più tardi, in Germania), in Italia, in Francia. Le élites politiche liberali costruttrici dello stato cercarono di frenare il potere della Chiesa e promossero attivamente l'anticlericalismo per tutta una serie di motivi. Questa offensiva fu di solito, anche se non sempre, centrata sull'istruzione e, ad un livello inferiore, sul diritto di famiglia. Furono introdotte riforme legislative con l'intento di sottrarre alla Chiesa gran parte del suo controllo sull'istruzione e sulla famiglia. Nel caso della Germania e dell'Italia questa offensiva assunse dimensioni anche maggiori, poiché si legò allo sviluppo dei movimenti per l'unità nazionale. In risposta a tutto ciò, la Chiesa, privata sempre più del tradizionale accesso privilegiato ai governi, dovette escogitare nuove strategie. Sebbene non gradisse la prospettiva, essa decise di adottare concreta- 
mente la strategia organizzativa, che consisteva nell'impiego delle risorse proprie della Chiesa in una lotta fuori dall'arena elettorale.

Le prime associazioni cattoliche sorsero, di solito ad opera di cattolici laici e sulla scia delle iniziali offensive anticlericali, in modo decentralizzato e non sistematico, spesso dopo aver ottenuto l'approvazione del vescovo locale. La materia prima di queste associazioni era costituita da parrocchie, club ricreativi, fratellanze, confraternite ed altri circoli religiosi che i cattolici avevano sviluppato soprattutto dopo la fine della rivoluzione francese (Mayeur 1980, 20). Per quanto già esistente, essa era tuttavia dispersa e decentralizzata. La decisione della Chiesa di intraprendere la strategia organizzativa fece sì che essa rilevasse la direzione di questi disparati gruppi cattolici, della loro espansione, della loro centralizzazione e del loro coordinamento in una rete di associazioni laiche; che stringesse il suo controllo su di esse e spostasse il centro d'interesse dei loro membri dalla vita privata alla vita pubblica. Le organizzazioni cattoliche erano adesso ufficialmente sponsorizzate dalla Chiesa e persero qualsiasi autonomia d'azione avuta in precedenza: il movimento cattolico era nato.

L'evidenza storica suggerisce che nella sua fase iniziale la strategia organizzativa fu concepita solo come misura di emergenza nel quadro di una reazione della Chiesa al liberalismo, all'anticlericalismo e alla scristianizzazione (Zeps 1979, 32; Gambasin 1969, 65). Le organizzazioni cattoliche sorsero spesso nel contesto della creazione di nuove reti di scuole cattoliche. Dunque, il focus di questa strategia fu la società civile piuttosto che lo stato o il processo elettorale, tanto che l'organizzazione fu implementata fuori dalla politica - e specialmente fuori dalla politica elettorale. Gli elementi che definirono la strategia organizzativa furono la subordinazione del movimento cattolico alla Chiesa e l'esclusione ufficiale della politica dalla sua azione. Per opporsi al rischio di una perdita di controllo sui suoi membri, la Chiesa inserì quelle organizzazioni all'interno di una struttura altamente centralizzata e gerarchica, con i vescovi al vertice. $\mathrm{La}$ «diretta dipendenza» delle associazioni dalla Chiesa accompagnò sempre, e in modo quasi automatico, ogni riferimento pubblico che di esse faceva la Chiesa stessa (Suardo 1962, 62; Bessières 1924). Dall'altro lato, la Chiesa impedì alle organizzazioni cattoliche l'azione politica: «creiamo associazioni cattoliche che si occupino solo delle questioni attinenti a Dio e alla Chiesa, 
escludendo rigorosamente la politica in modo da evitare qualsiasi tensione», scrisse l'arcivescovo di Vienna Rauscher ad un attivista cattolico (citato in Bled 1988, 122). Rauscher dichiarò ripetutamente che alle associazioni cattoliche non si sarebbe mai dovuto consentire di diventare associazioni politiche. Come affermò nel 1891 il sacerdote Bartolomeo Sandri, una delle figure di spicco della più importante organizzazione cattolica italiana, l'Opera dei Congressi, l'organizzazione doveva fare di tutto affinché «gli fosse impossibile entrare in contatto con la società liberale, col parlamentarismo, con le elezioni» (citato in Gambasin 1969, 85). Il motto calvinista olandese «la nostra forza sta nell'isolamento» incarnava il significato profondo di questa strategia.

La strategia organizzativa non riuscì a produrre risultati politici apprezzabili. Certamente non scoraggiò i liberali nelle loro offensive sempre più consistenti contro la Chiesa. Quest'ultima, da parte sua, a volte invitava i suoi membri a votare per candidati amici, ma, nel caso, lo faceva in modo decentralizzato, vago, disorganizzato, marginale e inefficace. In sostanza, la strategia organizzativa non ebbe alcun impatto politico, poiché non riuscì a trovar modo di rovesciare i governi liberali. In verità il suo obbiettivo di lungo termine era la riconquista dello stato dal basso, ma gli eventi dimostrarono che questa era una prospettiva veramente lontana, per non dire utopistica. La Chiesa fu dunque costretta a dedicarsi ai pericoli immediati legati all'intensificazione dell'offensiva anticlericale. Si prospettava una nuova scelta: entrare nell'arena elettorale o restarne fuori. La Chiesa fu molto riluttante ad intraprendere un'azione politica diretta. La sua rivendicazione di essere al di sopra delle parti fu compromessa e, al contempo, dato lo stretto controllo sulle organizzazioni cattoliche, la Chiesa ebbe serie difficoltà a contenere le pressioni degli attivisti cattolici laici per una maggiore autonomia. Zerbi annota che all'interno dell'Opera dei Congressi italiana i leader laici finirono «per dare le direttive ai vescovi, provocando frequenti lamentele nell'episcopato» (1961, 78). Le parole del vescovo italiano mons. Scalabrini sono stridenti: «spero che il tempo dei vescovi col galero sia finalmente giunto al termine!» (citato in Zerbi 1961, 79). Ci si attendeva che la partecipazione diretta al processo elettorale aggravasse questa tendenza. D'altro canto, però, la realtà delle offensive anticlericali costrinse la Chiesa a compiere una scelta, qualunque ne fosse il costo. Riluttante e con grande esitazione, la Chiesa deci- 
se di praticare la strategia «partecipativa», nonostante i suoi notevoli costi. Volendo evitare di trasformarsi essa stessa in partito politico, la Chiesa mise insieme ampie ma fragili coalizioni di gruppi conservatori. Queste coalizioni si battevano nelle campagne elettorali all'insegna della difesa della Chiesa. Diversamente dalla precedente prassi di un appoggio non sistematico ai candidati conservatori, questa strategia comportò un'azione elettorale coordinata, soprattutto grazie alle organizzazioni di massa della Chiesa di recente costituzione. Un tale appoggio richiese la trasformazione di queste formazioni in organizzazioni sempre più orientate alla politica e alle elezioni, in modo da aiutare così quelle coalizioni ad affrontare le battaglie elettorali e ad indurre $i$ liberali ad attenuare l'anticlericalismo. Tuttavia, la politicizzazione del movimento cattolico non implicò un'autonomia d'azione. Come ha sottolineato in modo eloquente De Rosa in riferimento all'Italia, «i moderati entravano solo come eletti ed i cattolici solo come elettori» $(1972,266)$.

L'impiego elettorale delle organizzazioni cattoliche di massa doveva essere temporaneo e non doveva condurre alla formazione di un partito. Allo stesso tempo, però, la Chiesa si aspettava che queste coalizioni elettorali avrebbero prima o poi smesso di identificarsi esclusivamente con essa. L'obbiettivo e l'esito atteso della strategia partecipativa era soltanto la revoca delle riforme anticlericali e la loro sostituzione con un nuovo «concordato» interno. La logica conclusione della strategia partecipativa della Chiesa era la sottrazione del fattore cattolico dalla vita politica dei paesi (Anderson 1981, 194). Dunque, l'obbiettivo ultimo di questa strategia era un ritorno al passato piuttosto che l'istituzionalizzazione del cattolicesimo politico. Si pensava che, non appena il pericolo dell'anticlericalismo fosse diminuito, le organizzazioni cattoliche sarebbero tornate ai loro compiti originali, che nulla avevano a che fare con le elezioni, e sarebbero rimaste fuori dalla politica, sotto lo stretto controllo della Chiesa ${ }^{14}$. Come Gramsci ha sottolineato nella sua analisi

14 Questo era stato il destino delle organizzazioni cattoliche di più antica data, quali l'austriaca Katholikenverein e la tedesca Piusverein, le quali furono depoliticizzate dopo che il pericolo delle rivoluzioni del 1848 era venuto meno. Questo è stato anche il destino finale del movimento cattolico contemporaneo: oggi l'«Azione Cattolica» è costituita di organizzazioni apolitiche di cattolici laici, strettamente controllate dalla Chiesa. Questo progetto fu infatti promosso dopo la formazione dei partiti confessionali con l'enciclica papale Ubi arcano Dei del 1922. La nozione di subordinazione laica costituì la base di un nuovo concetto, il «mandato» (Aubert 1982, 202). L'enciclica definì 
del movimento cattolico, per la Chiesa le organizzazioni laiche di massa dovevano «di norma svolgere un ruolo congiunturale od occasionale, disṕensabile in caso di necessità» (Fulton 1987, 212). Vi è più di una conferma storica alla circostanza che la Chiesa non intendeva politicizzare stabilmente le organizzazioni cattoliche, né progettava di fonderle insieme agli alleati politici all'interno di un unico partito confessionale ${ }^{15}$. In sintesi, come sostiene Suardo a proposito dell'Italia, all'inizio del XX secolo «in Vaticano c'è un'avversione assoluta a che un partito di cattolici esista o sembri esistere» $(1962,135)$. E non solo la Chiesa non fu capace di prevedere l'emergere di partiti confessionali, ma fece tutto ciò che era in suo potere per impedire a quella prospettiva di tradursi in realtà. All'inizio la Chiesa prese numerose misure preventive che rafforzarono il suo controllo sul personale e sui membri (Jemolo 1960). Quando queste misure si rivelarono insufficienti, la Chiesa pose un freno ad ogni tentativo diretto a sviluppare l'indipendenza, l'autonomia o la politicizzazione non controllata delle organizzazioni cattoliche. La repressione fu comunque più semplice al tempo della strategia organizzativa. La dinamica creata dall'offensiva anticlericale e dalla strategia partecipativa innalzò i costi della repressione e non permise alla Chiesa di disporre a proprio piacimento delle organizzazioni cattoliche. Di conseguenza, la Chiesa tentò di ostacolare qualsiasi iniziativa orientata alla formazione di organizzazioni cattoliche indipendenti e di partiti confessionali. L'opera di repressione e di ostruzione si verificò in tutti i casi presi in esame e fu centrale nella strategia e nell'azione della Chiesa.

Allo stesso modo, anche le élites politiche conservatrici non volevano né progettavano la formazione di partiti confessionali. «La destra parlamentare [belga], cattolica e liberale, rigettò l'idea di un partito confessionale organizzato e centralizzato» sintetizza Soete $(1984,201)$. I conservatori vedevano l'identificazione con la Chiesa e la promozione della religione come una strategia temporanea. Come dichiarò nel 1872 il tedesco Lud-

l'Azione Cattolica come «la partecipazione del laicato cattolico all'apostolato della gerarchia, per la difesa dei principi religiosi e morali, per lo sviluppo di un'azione sociale sana e utile, sotto la guida della gerarchia ecclesiastica, al di fuori e al di là di tutti i partiti politici, al fine di restaurare la vita cattolica nella famiglia e nella società».

15 Per i riferimenti dai quali emerge l'evidenza che la Chiesa non gradiva, non voleva, ed anzi ostacolò la formazione di partiti confessionali si rinvia a Kalyvas (1996). 
wig Windthorst, «se i lamenti eccessivi dei cattolici si sono finalmente placati [...] il Zentrum avrà il piacere di dissolversi» (citato in Anderson 1981, 217). Il carattere provvisorio di queste alleanze fu spesso manifestato apertamente. Ad esempio, un accordo elettorale locale tra conservatori e cattolici in Italia affermava esplicitamente che la lista comune di candidati era $o c$ casionale (Suardo 1962, 103-104). Il carattere provvisorio fu sottolineato inoltre dalla circostanza che queste coalizioni soffrirono di molteplici antagonismi, dal momento che le loro componenti avevano priorità molto diverse e finanche antitetiche. In Belgio, per esempio, il tentativo delle organizzazioni cattoliche di imporre alla destra un programma cattolico rigoroso e vincolante, che divenne noto come programmisme, provocò una violenta reazione della stessa destra (Preneel 1982, 124). I conservatori si aspettavano che la possibile introduzione nel futuro di alcune misure anticlericali o il ricordo dell'offensiva anticlericale dei liberali avrebbe legato stabilmente il voto dei cattolici ai loro partiti ${ }^{16}$.

Dunque, sia la Chiesa che i conservatori consideravano il successo a $\mathrm{t} 3$ uguale ad un eventuale ritorno a $\mathrm{t} 1$, sebbene a condizioni nuove e favorevoli. La Chiesa avrebbe mantenuto la maggior parte dei suoi privilegi e impiegato le sue organizzazioni per prevenire future offensive contro di essa, mentre i conservatori avrebbero acquisito la fedeltà di una quota notevole di elettori, in precedenza non mobilitati o divisi lungo altri cleavages, senza essersi esposti alle spese e ai rischi del processo di costruzione di un'organizzazione di massa.

Dati questi vincoli, come fecero ad emergere partiti confessionali basati sulle organizzazioni cattoliche di massa? Come riuscirono ad affermarsi come partiti cattolici e a dominare lo spazio politico non occupato dai socialisti? Come fu possibile conseguire un risultato così inefficiente (per ambedue gli attori centrali)? La risposta è che le strategie adottate dalla Chiesa ebbero conseguenze non volute e tuttavia importanti per l'azione collettiva e la formazione di un'identità. Queste strategie mobilitarono politicamente ed elettoralmente, per la prima volta, i cattolici laici in quanto cattolici, creando effettivamente un'iden-

16 Ciò è quanto accadde infatti in Francia. Gli elettori religiosi hanno sempre dato il loro appoggio alla destra. La religione fu, e rimane ancora, un eccellente fattore di previsione del comportamento elettorale dei francesi (Boy e Meyer 1993; Derivry e Dogan 1971). 
tità politica cattolica. Per questo nuovo attore politico, gli attivisti cattolici ${ }^{17}$, la formazione dei partiti confessionali fu tutt'altro che inefficiente: li legittimò, li emancipò dal controllo della Chiesa e consentì loro di promuovere la loro agenda politica, che, come la Chiesa aveva giustamente temuto, fu spesso distinta dalla sua: così come Josef Wackernell, il leader dei conservatori tirolesi, ebbe a lamentarsi nel 1903 del Partito Cristiano Sociale austriaco, per questi partiti «vale soltanto la volontà della gente, non l'autorità ecclesiastica» (citato in Boyer 1995, 95); e, ancora, come dichiarò il sacerdote tedesco Eduard Cronenberg nel 1871: «gli interessi dei cattolici si estendono molto al di là degli interessi della Chiesa cattolica» (citato in Brose 1985, 47).

La creazione di organizzazioni cattoliche di massa e la loro partecipazione inizialmente non autonoma alla politica elettorale, comunque, non garantirono di per sé la loro trasformazione in partiti confessionali, dato che sia alla Chiesa che ai conservatori una tale prospettiva non piaceva proprio. Ciò che rese possibile la transizione dalla strategia partecipativa alla formazione di partiti confessionali (in altre parole, la traduzione della nuova identità politica in un nuovo partito) fu il successo elettorale ampio e inaspettato delle coalizioni che combatterono campagne «in difesa della Chiesa». Per gli attivisti cattolici il successo elettorale fu la prova che la religione era una questione politica estremamente vantaggiosa, con un potenziale di sfruttamento di forze enormi. Insieme con i partiti socialisti, e comunque prima di loro, i movimenti cattolici furono pertanto i vincitori della politica di massa. Oltretutto, il successo elettorale rivelò anche una nuova ed ampia fonte di potere e di legittimazione che avrebbe sostituito la Chiesa: gli elettori. Fornendo loro questa nuova fonte di potere e di legittimazione, il successo elettorale dette agli attivisti cattolici l'opportunità unica di condurre a termine, contro i desideri della Chiesa e dei conservatori, la trasformazione delle effimere e disomogenee coalizioni a favore della Chiesa in partiti politici. Il successo elettorale fu dunque il punto di svolta nel processo di formazione dei partiti confessio-

17 È importante sottolineare che se i cattolici laici erano già fisicamente presenti nei periodi $t 1$ e $t 2$, essi non rappresentavano ancora un attore politico. E la loro mobilitazione come cattolici che li trasforma in attore politico. Bisogna inoltre aggiungere che se è vero che la maggioranza degli attivisti cattolici era costituita di laici, è anche vero che, almeno in un primo tempo, una parte importante era rappresentata da membri del basso clero. 
nali. È impossibile sottolineare l'importanza del successo elettorale meglio di quanto fece un belga di quel tempo: «fino al 1884 [il partito] si chiamava "partito conservatore". Da allora si chiama "partito cattolico"» (Carton de Wiatr in Guyot de Mishaegen 1946, III; il 1884 fu l'anno della vittoria dei cattolici).

L'affermazione elettorale delle coalizioni pro-Chiesa fu rimarchevole (tab. 2). In due casi - Belgio 1884 e Olanda 1888 queste coalizioni vinsero in modo clamoroso. Anche quando non ottennero la maggioranza dei voti, queste coalizioni fecero un ingresso di grande effetto nella politica dei partiti. Ciò che contava, infatti, non era semplicemente il risultato elettorale la percentuale di voti ricevuti o il numero di seggi vinti. Le percentuali conseguite dalle coalizioni cattoliche in Germania o in Italia non furono certamente alte in termini assoluti. Il Zentrum ottenne il $18,7 \%$ dei voti validi nel 1871 , mentre il Partito Popolare Italiano prese il 20,5\% nel 1919. La cosa importante fu piuttosto la diffusa percezione di tutti i partecipanti che il risultato conseguito dalle coalizioni favorevoli alla Chiesa costituisse un grande successo elettorale (Lynch 1993, 34; Agócs 1988, 14; Anderson 1986, 95-96; Molony 1977, 67). Questa percezione derivava da tre ragioni. Primo, il piazzamento relativo dei partiti cattolici fu alto. Sia il Zentrum che il PPI divennero il secondo partito nei rispettivi paesi. Secondo, le perdite che essi inflissero ai liberali furono notevoli ${ }^{18}$. Terzo, questa fu la prima dimostrazione delle forze politiche organizzate favorevoli ai cattolici nella lotta a difesa della Chiesa. Di conseguenza, anche se non sempre alto in termini assoluti, il risultato elettorale delle coalizioni cattoliche fu interpretato come un indice del loro straordinario potenziale futuro.

Impressionante non fu soltanto l'affermazione elettorale, ma anche il fatto che essa venne a sorpresa. Nessuno si aspettava che le coalizioni cattoliche avrebbero ottenuto un risultato così importante. Nei commenti degli osservatori, dei partecipanti e degli storici del tempo, in tutti i casi in esame, si registra un ele-

18 In Italia, le fazioni conservatrici, liberali e democratiche ottennero il $36 \%$ dei suffragi alle elezioni del 1919 quando il Partito Popolare fece la sua prima apparizione, scendendo dal 61\% delle precedenti elezioni del 1913. Analogalmente, in Germania tutti i 25 deputati cattolici, eletti in collegi fondamentalmente cattolici, che erano stati fedeli a partiti anticlericali persero i loro seggi alle elezioni del 1871. Come afferma Reinermann, il risultato del Zentrum nel 1871 fu «una chiara sconfitta per Bismarck» $(1993,762)$. 
ТАВ. 2. Le prime affermazioni elettorali delle coalizioni antiliberali

\begin{tabular}{|c|c|c|}
\hline Paese & Anno & Risultati \\
\hline Belgio & 1884 & $\begin{array}{l}\text { Cattolici: } 86 \text { seggi } \\
\text { Liberali: } 52 \text { seggi }\end{array}$ \\
\hline Olanda & 1888 & $\begin{array}{l}\text { Cattolici e Calvinisti: } 54 \text { seggi } \\
\text { Liberali: } 44 \text { seggi }\end{array}$ \\
\hline Austria & $\begin{array}{l}1887 \\
1895\end{array}$ & $\begin{array}{l}\text { [elezioni locali] } \\
\text { [elezioni municipali, Vienna] }\end{array}$ \\
\hline Germania & 1871 & $\begin{array}{l}\text { Cattolici: } 61 \text { seggi } \\
\text { Liberali: } 125 \text { seggi }\end{array}$ \\
\hline Italia & $\begin{array}{l}1913 \\
1919\end{array}$ & $\begin{array}{l}\text { Patto Gentiloni ( } 228 \text { Liberali su } 310 \text { eletti } \\
\text { con i voti dei Cattolici) } \\
\text { Cattolici: } 100 \text { seggi } \\
\text { Liberali (varie frazioni): } 197 \\
\text { Socialisti: } 156 \text { seggi }\end{array}$ \\
\hline
\end{tabular}

mento di sorpresa (Anderson 1986, 93-96 e Anderson 1981, 116; Canavero 1981, 290; Malgeri 1969, 115; Windell 1954, 127 e 282; Pirenne 1932, 297-298; Woeste 1927, 216). La circostanza che il successo elettorale colse tutti di sorpresa è cruciale. È proprio la mancata previsione di questi successi elettorali che spiega la sottovalutazione da parte della Chiesa e dei conservatori della loro possibile perdita di controllo sulle organizzazioni cattoliche e, pertanto, della probabilità di formazione di partiti confessionali. Questa mancata previsione fu dovuta a due fattori. Il primo è che il potenziale elettorale della religione come questione politica dominante, soprattutto dopo l'avvento dell'offensiva anticlericale, non era stato esattamente valutato. Il secondo è che quello era un periodo di transizione verso una nuova epoca politica: quella della politica di massa. Il potere dell'organizzazione di massa o anche gli effetti del suffragio maschile allargato o universale erano sconosciuti. Il loro avvento rappresentò «uno sbalorditivo salto nel buio» (Jones 1985, 233). Democrazia e incertezza andavano davvero mano nella mano. Come ha sottolineato Przeworski, la democrazia è «l'istituzionalizzazione dell'incertezza»: «gli esiti che sono improbabili possono accadere ed accadono ${ }^{19}(1986,58)$.

19 A questo punto è necessario porsi una domanda in riferimento alla diffusione delle informazioni tra i paesi. Con le sconfitte dei liberali e le vittorie dei cattolici in tutta Europa, come poté prevalere ancora una tale miopia? $\mathrm{Ci}$ sono due risposte. La prima, il panorama politico in Europa era tutt'altro che chiaro. Il caso della Francia, dove, senza soluzione di continuità tra il 1879 e il 1919, i repubblicani anticlericali con- 
La Chiesa cadde quindi vittima del successo della propria strategia: non poteva trasformarsi essa stessa in un partito politico in modo da controllare questi sviluppi, né poteva congelare il processo, impedire che le sue organizzazioni di massa evolvessero in partiti politici e ricondurre all'ovile gli attivisti cattolici. Avendo appena conquistato il potere politico, quegli attivisti non erano più disposti ad accettare di essere relegati ai margini della vita politica - spettatori passivi sottoposti agli ordini della gerarchia ecclesiastica. Non appena si rese conto che questi partiti confessionali emergenti stavano amputando una gran parte delle sue organizzazioni di massa e del suo personale, e che stavano riducendo il suo controllo sul «mondo cattolico», la Chiesa non ebbe altra scelta che riconoscerli. Essa non poteva permettersi di reprimerli a causa della nuova e notevole legittimazione popolare che questi partiti avevano acquisito con lo sbalorditivo esito elettorale. Come Molony riassume in riferimento all'Italia, «il Vaticano acconsentì alla formazione del PPI [il partito cattolico italiano] perché [...] non poté impedirlo» $(1977,6)$. Questo riconoscimento fu seguito ancora da un vigoroso tentativo di recuperare i suoi membri dai nuovi partiti, soprattutto mediante lo sviluppo di nuove organizzazioni di massa dell'Azione Cattolica (si veda la nota 14). La storia dei partiti confessionali è piena di conflitti con la Chiesa. Tant'è che, quando le circostanze politiche furono favorevoli, la Chiesa contribuì senza esitazioni alla distruzione di questi partiti. Ne sono esempio il Partito Popolare Italiano tra il 1923 e il 1926, e il Zentrum tedesco e il Partito Cristiano Sociale austriaco nel 1934 (Gellott 1987). Creando senza volerlo una nuova classe politica, la strategia della Chiesa influenzò inoltre anche l'altro attore. I conservatori, che guidarono le coalizioni elettorali della

tinuarono ad attaccare la Chiesa e a sconfiggere i conservatori filo-cattolici, costituiva un esempio manifesto. La vittoria dei repubblicani nelle elezioni francesi del 1879 , ad esempio, fu interpretata dai liberali belgi come un successo del liberalismo sull'ultramontanismo e rafforzò la loro convinzione che l'offensiva contro la Chiesa nel campo dell'educazione fosse la strada giusta da percorrere. La seconda risposta è che, in assenza di un modello comune, i casi particolari furono spesso interpretati come idiosincratici e non suscettibili di generalizzazione. Ad esempio, la circostanza che le prime affermazioni dei cattolici si verificarono in Germania fu spesso associata alla situazione peculiare dei cattolici tedeschi in quanto minoranza. Quando il giornale liberale austriaco «Neue Freie Presse» riportò nel 1868 la notizia della vittoria di un candidato filo-cattolico in Francia, non fu tracciato nessun parallelo con l'Austria. Mentre, allorché il giornale filo-cattolico austriaco «Das Vaterland» menzionò nel 1868 i successi elettorali dei cattolici nella Germania meridionale, ciò fu tutt'altro che ignorato. 
strategia partecipativa, furono ben presto spazzati via dai leader delle organizzazioni cattoliche di massa.

\section{La mancata formazione di un partito confessionale in Francia}

In Francia non emerse alcun partito confessionale. Come confermano Boutry e Michel, «la vita politica francese non ha mai sperimentato un vero partito confessionale, ossia un'organizzazione politica con degli iscritti, un elettorato, un programma e degli obbiettivi dipendenti da una confessione» (1992, 673). I medesimi autori sottolineano la rilevanza di questo fatto affermando che «la non esistenza di un partito confessionale e il fallimento di ogni formula che perseguisse quest'obbiettivo costituiscono un reale problema storico» ${ }^{20}(1992,664)$.

Questo fallimento costituisce un serio problema per la teoria di Lipset e Rokkan, poiché essi predicono la formazione di partiti confessionali quando emergono cleavages religiosi, come fu nel caso della Francia. Gli autori più che affrontare il problema lo eludono, facendo un vago riferimento alle forze della storia ed affermando che i partiti conservatori francesi furono «implicitamente» dei partiti confessionali ${ }^{21}$ (Lipset e Rokkan 1967, 40 e 34). Oltretutto, la mancata formazione di un partito confessionale in Francia è di per sé particolarmente sconcertante,

20 La maggior parte dei lavori sul tema si focalizzano, curiosamente, sul fallimento del MRP nel dopoguerra. Se è vero che ciò è di per sé un'importante questione, è altrettanto vero che il mancato emergere di un partito confessionale durante la III Repubblica, l'epoca della formazione dei cleavages e la cristallizzazione del sistema partitico rappresentano problemi teorici fondamentali.

21 L'argomento che i conservatori francesi fossero implicitamente confessionali non può essere considerato valido. Mayeur $(1980,9)$ sottolinea che i partiti monarchici francesi, anche se appoggiati dai cattolici e difensori degli «interessi religiosi», costituiscono un fenomeno del tutto diverso dai partiti confessionali. Il processo di formazione partitica conta come e più del comportamento elettorale e del cleavage. Di più, la formazione partitica definisce il modo nel quale i cleavages diventano politicizzati e modellano i rapporti politici. Dopo che i partiti che rappresentano un determinato cleavage sono sorti, o «dopo che sono divenuti stabili e organizzativamente istituzionalizzati», allora «essi sviluppano la loro forza autonoma e, a sua volta, cominciano ad agire influenzando la vita sociale, culturale e politica» (Bartolini e Mair 1990, 218). In altre parole, un cleavage religioso senza un partito confessionale produrrà un ambiente politico significativamente diverso da un cleavage religioso con un partito confessionale. L'esempio seguente illustra questo punto. I partiti confessionali (ma non i partiti conservatori appoggiati dagli elettori religiosi) furono costretti ad assorbire le organizzazioni degli operai che erano state formate in precedenza dalla Chiesa; questa circostanza ebbe importanti effetti sia nei rapporti politici che nelle politiche pubbliche. 
dal momento che la Francia era un paese nel quale il cattolicesimo fu tanto forte ed influente che essa era chiamata la «figlia maggiore» della Chiesa, e nel quale il conflitto tra stato e Chiesa, soprattutto tra 1879 e 1906, più che intenso fu violento: tanto che proprio il conflitto stato-Chiesa divenne la questione ideologica chiave della III Repubblica. Il caso della Francia rappresenta, infine, un test cruciale per il modello di formazione partitica sviluppato in questo articolo. Il modello deve spiegare, senza modificazioni analitiche, ambedue i casi di formazione e di non formazione. Una spiegazione egualmente coerente ed integrata, che proceda a partire dalle stesse assunzioni ed impieghi le stesse variabili, deve essere fornita per ciascuno degli esiti (Luebbert 1991, 5). In questa sede, offro una sintesi del trattamento del caso francese che ho sviluppato in dettaglio altrove (Kalyvas 1996).

Le spiegazioni della mancata formazione di un partito confessionale in Francia variano dall'eccezionalità francese ad argomenti strutturali sul grado di scristianizzazione in Francia. Non vi è comunque evidenza che la Francia fosse a quel tempo meno religiosa del resto dell'Europa, né che essa vivesse un declino secolare della religiosità. Secondo l'interpretazione più recente, la causa fondamentale della mancata formazione di un partito confessionale in Francia fu la non volontà a farlo dei principali attori: Boutry e Michel affermano che «la prima causa di questa assenza deve essere ricercata nel rifiuto degli attori cattolici, laici e clero, di creare un tal partito in Francia» (1992, 673). Questi autori si soffermano particolarmente sul ruolo della gerarchia ecclesiastica: «l'atteggiamento della gerarchia cattolica è spiegato in larga misura dalla volontà di mantenere chiaramente distinti la causa e gli interessi della Chiesa da qualsiasi movimento politico» $(1992,676)$. Tuttavia, l'analisi comparata degli altri cinque casi ha dimostrato che ciò fu vero ovunque: $\mathrm{i}$ due attori principali - la Chiesa e i conservatori - certamente non desiderarono e non promossero la formazione di partiti confessionali in alcun paese.

La risposta più immediata al caso francese risiede nei ripetuti fallimenti elettorali delle coalizioni che combatterono a difesa della Chiesa: dal 1876 al 1919 la destra perse tutte le competizioni elettorali e rimase all'opposizione. Queste sconfitte elettorali vennero interpretate come la prova definitiva che la religione fosse una issue perdente per la destra. Senza affermazione elettorale il modello predice che non possa essere formato 
alcun partito. $\mathrm{Ma}$ questa risposta pone immediatamente un nuovo interrogativo: perché queste coalizioni furono così perdenti in Francia? La risposta è che la destra in Francia era divisa e disorganizzata. In elezioni cruciali numerosi candidati conservatori combatterono l'uno contro l'altro, mentre la massa dei potenziali elettori cattolici rimaneva sottomobilitata, come testimoniato dagli alti tassi di astensionismo nelle regioni tradizionalmente cattoliche (Mayeur 1973, 102). Come spiegarlo? La destra in Francia non riuscì a mobilitare i cattolici perché la Chiesa scelse di non praticare la strategia organizzativa. L'assenza di organizzazioni cattoliche di massa in Francia è un fatto riscontrato empiricamente da molti studi storici. Come fa notare Poulat $(1977,130)$, la «dimensione popolare» del cattolicesimo era assente in Francia se la si confronta al Belgio, alla Germania, all'Italia. La Chiesa fu contraria alla creazione di organizzazioni di massa perché, quando affrontò la prima ondata dell'offensiva anticlericale nel 1879 , conservò la seria speranza che $\mathrm{i}$ repubblicani anticlericali sarebbero stati rovesciati dalla destra. La fonte di questa valutazione era la forza relativa della destra francese (diversamente da quella della destra di altri paesi), l'instabilità ampiamente percepita del regime repubblicano e la connessione tra il regime alternativo, la monarchia, e la protezione della Chiesa. In altre parole, la plausibilità del rovesciamento del regime repubblicano influì sulla struttura dei costi di opportunità della Chiesa: il costo della strategia organizzativa eccedeva a quel punto il costo dell'attesa dell'estromissione dei repubblicani e della restaurazione della monarchia mediante gli abituali mezzi elettorali o persino con un colpo militare: al tempo t1, NF è preferito ad $O$ (fig. 3 ).

$\mathrm{E}$ importante vedere quanto fosse differente, sotto questo aspetto, la situazione francese da quelle prevalenti negli altri cinque paesi. In questi ultimi, i regimi associati alle offensive anticlericali erano granitici: non esisteva alcuna speranza di un loro rovesciamento e di un conseguente avvento di un regime apertamente favorevole alla Chiesa. Così come in Francia, in Germania e in Italia i cattolici nutrivano sentimenti di sdegno e di rifiuto nei confronti dei regimi dominanti, ma la sconfitta militare li aveva lasciati privi della speranza di un loro rovesciamento. In Italia il Vaticano fu sconfitto militarmente nel 1862 e nel 1870 , mentre la sconfitta militare dell'Austria ad opera della Prussia nel 1866 equivalse ad una sconfitta del cattolicesimo tedesco e la conseguente unificazione della Germania trasformò i 


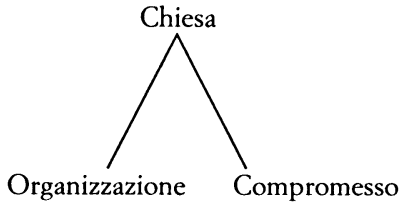

t1
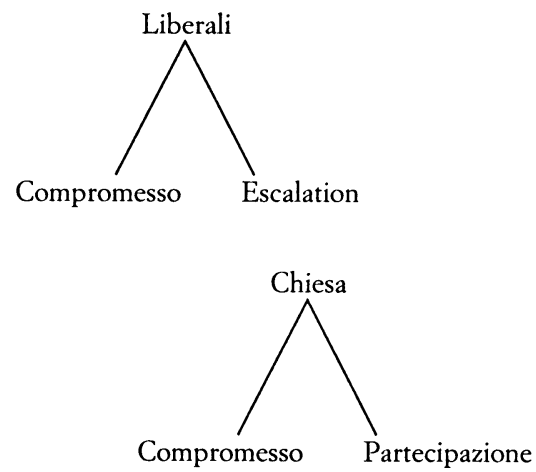

t2

Natura del risultato elettorale

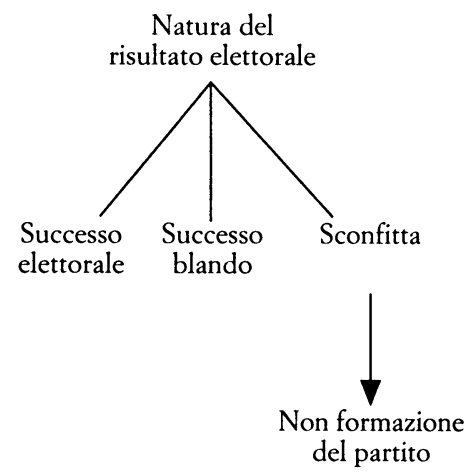

FIG. 3. Il fallimento del processo di formazione di un partito confessionale (Francia).

cattolici in una minoranza. In Olanda (dove i cattolici erano stati sin dall'inizio una minoranza), in Austria e in Belgio imperatori e re non mostrarono alcuna volontà di bloccare le offensive anticlericali dei liberali, né mandarono segnali per cui avrebbero preso in considerazione un simile obbiettivo. Al contrario, essi dettero la prova di voler collaborare con loro (Stengers 1983, 388). La mancanza di opzioni alternative, soprattutto 
istituzionali, rese $\mathrm{i}$ costi dell'organizzazione accettabili per la Chiesa.

Quando fallì il colpo di stato Boulanger a favore della monarchia nel 1889, la Chiesa capì finalmente che aveva scommesso sul cavallo sbagliato e che la Repubblica sarebbe rimasta. A quel punto, data la gravità dell'intensificazione delle offensive anticlericali e le perdite accumulate, l'alternativa rimanente era una rapida implementazione della strategia partecipativa: la creazione di una destra unificata che avesse come obbiettivo fondamentale la difesa della Chiesa (piuttosto che la restaurazione della monarchia). Questo passo fu compiuto nel 1892, in ciò che divenne noto come il Ralliement (Sedgwick 1965). La creazione di organizzazioni di massa fu quindi pressoché scavalcata, a causa del tempo che avrebbe richiesto e della circostanza che il mercato dell'organizzazione era già stato accaparrato dai competitori della Chiesa. Numerosi studi storici (soprattutto di politica locale) hanno dimostrato che i repubblicani prima e i radicali dopo si rivelarono i più pronti alla politica di massa, creando un'organizzazione di gran lunga migliore di quella della destra o della Chiesa, persino nelle regioni considerate tradizionalmente cattoliche (Huard 1982, 226 e 331-332; Rivet 1979, 440). Quando, nel 1902, fu formata la più forte coalizione procattolica con il nome di Action Liberale Populaire (ALP) era troppo tardi. Senza un'organizzazione di massa, questa coalizione non poteva che essere sconfitta: il tasso di mobilitazione dei potenziali elettori cattolici rimase basso, mentre molti contadini religiosi appoggiarono i partiti repubblicani. L'effetto cumulato dell'assenza di un'organizzazione, secondo un osservatore del tempo, fu quello di spingere i potenziali attivisti cattolici in uno stato di «indolente passività che costò loro cara» (Viance 1930, 15). L'alienazione dei cattolici crebbe con queste sconfitte e, diversamente dagli altri paesi, fece sì che essi non riuscissero ad integrarsi nella Repubblica, come dimostrato dal fatto che furono materia prima di reclutamento di movimenti antidemocratici quali l'Action Française. Nel 1906, a seguito dell'approvazione della legge sulla separazione tra stato e Chiesa e di un nuovo (e ultimo) insuccesso elettorale dell'ALP, l'offensiva anticlericale cessò e la Chiesa ammise la sconfitta. 


\section{Conclusioni}

La formazione dei partiti confessionali si è compiuta in tre tappe. In primo luogo, la formazione delle organizzazioni cattoliche di massa (la strategia organizzativa) sparse il seme di un'identità cattolica e creò attivisti laici e del basso clero in organizzazioni strettamente controllate dalla Chiesa ed operanti al di fuori dell'arena elettorale. In una seconda fase, l'ingresso della Chiesa nell'arena elettorale e la creazione di coalizioni proChiesa (la strategia partecipativa) politicizzò questa identità embrionale trasformando le organizzazioni cattoliche in macchine politiche. Infine, l'affermazione elettorale di queste coalizioni consentì agli attivisti cattolici di costituire con successo, nonostante le intenzioni della Chiesa e dei conservatori, partiti confessionali. L'identità politica cattolica fu costruita dopo che si formarono i partiti confessionali: come nota Bourdieu, la costruzione di gruppi mobilitati o «mobilitabili» richiede «l'istituzionalizzazione di un'organizzazione stabile capace di rappresentarli» $(1987,13)$.

Tutte queste tre tappe sono necessarie affinché emerga un partito confessionale. La creazione di un'identità cattolica embrionale mediante l'organizzazione di massa fu necessaria ma non sufficiente alla formazione di una distinta identità politica cattolica. Se il movimento cattolico, per usare l'espressione di Traniello, «conteneva nel suo grembo, forse sin dalle origini, un embrione di partito» $(1982,46)$, ciò che portò alla sua nascita fu la strategia partecipativa. La sola organizzazione di massa poteva essere tenuta sotto controllo; era necessario che le fossero forniti nuovi sbocchi e arene alternative affinché diventasse politicizzata e, alla fine, autonoma. E solo la partecipazione politica poteva renderlo possibile. D'altro canto, se la strategia partecipativa contribuì alla creazione di un'identità politica cattolica e determinò un'azione collettiva, essa non avrebbe potuto però condurre alla formazione di partiti confessionali senza una precedente implementazione della strategia organizzativa. In primo luogo, fu la strategia organizzativa che rese la strategia partecipativa elettoralmente efficace; e, in secondo luogo, anche se la strategia partecipativa avesse determinato delle affermazioni elettorali senza una precedente organizzazione di massa, i conservatori a sé stanti avrebbero mancato di legittimazione, di credibilità e, soprattutto, degli incentivi per appropriarsi della religione su base permanente al fine di costruire un partito. Ciò fu 
possibile solo attraverso l'azione degli attivisti cattolici, membri delle organizzazioni di massa. Infine, organizzazione e partecipazione senza successo elettorale non avrebbero portato da nessuna parte. La religione avrebbe dimostrato di essere una issue perdente, ogni sforzo di costruire un partito politico cattolico non avrebbe potuto che fallire, e tutti gli attori rilevanti avrebbero cercato strategie e issues alternative.

Questa è, dunque, una storia di conseguenze impreviste ed indesiderate dell'azione politica razionale (Boudon 1982). Quando i liberali lanciarono la loro offensiva anticlericale, nessuno dei principali attori politici desiderava, attendeva o progettava la creazione di partiti confessionali. L'offensiva dei liberali contro la Chiesa, per usare le parole di Sewell, «scatenò una catena imprevedibile e sempre più densa di scontri che culminò in un grande e durevole cambiamento delle identità collettive» $(1992,15)$. Le offensive anticlericali furono tuttavia un elemento necessario ma non sufficiente per la formazione dei partiti confessionali. Dove non si verificò alcuna offensiva, come nel caso dell'Irlanda o della Spagna post-franchista, non si formarono partiti confessionali. Ma le offensive anticlericali non si tradussero sempre o necessariamente nella formazione di tali partiti, come evidenziato dal caso francese. Piuttosto che fattore causale della formazione di partiti confessionali, le offensive anticlericali furono una molla che impose dei vincoli alle strategie degli attori principali e che strutturò le loro opzioni lasciando spazio alla modificazione delle strategie che furono adottate. Le «giunture critiche» non sempre producono cleavages e ciò spiega perché è essenziale specificare i microfondamenti della teoria dei cleavages di Lipset e Rokkan. L'emergere di un'identità politica cattolica non fu né automatica, né naturale; fu l'esito contingente di conflitti tra una varietà di attori sotto vincoli specifici e può essere compresa solo mediante la disamina delle azioni e delle scelte degli attori principali e mediante gli esiti che queste azioni determinarono.

Il processo di formazione partitica ebbe importanti implicazioni politiche e sociali. I nuovi partiti furono socialmente eterogenei poiché nacquero sulla base di un richiamo religioso: l'«interclassismo» fu un effetto del processo di formazione partitica piuttosto che un'ideologia soltanto. Questi partiti occuparono lo spazio «conservatore» o «non-socialista» nello spettro politico; e, tuttavia, furono cosa diversa dai tradizionali partiti conservatori. Essi dovettero, ad esempio, occuparsi delle conse- 
guenze dell'assorbimento delle organizzazioni operaie che la Chiesa aveva costituito nel contesto della strategia organizzati$\mathrm{va}^{22}$. Oltretutto, non poterono liberarsi del loro carattere confessionale, perché la religione era diventata il fondamento dell'identità politica dei loro membri e il cemento che teneva insieme la loro eterogenea base sociale. Questi partiti, di conseguenza, dovettero mantenere un legame con la Chiesa e la religione. $\mathrm{Ma}$, essendo nati nonostante le intenzioni della Chiesa, essi si risentirono dei suoi continui tentativi di controllarli e di sottometterli e riuscirono pertanto a declericalizzarsi. Negli anni seguenti alla loro formazione, questi partiti ampliarono le distanze dalla Chiesa e spostarono le loro priorità politiche dalle questioni clericali. Con ciò essi «desacralizzarono», per usare il felice termine di Formigoni (1988, 28), la politica dei loro paesi. Mantennero un riferimento ideologico alla religione, ma ridefinirono il significato della religione in funzione della politica, trasformando il cattolicesimo in un astratto concetto morale e umanitario controllato e mediato da loro più che dalla Chiesa. Come osserva Lorwin, questo concetto fu «tanto vago quanto dettagliata e specifica fu la dottrina della Chiesa» (1971, 168169). I partiti confessionali poterono, in effetti, affermare di essere allo stesso tempo «cattolici» e secolari. Negli anni questo sviluppo assunse dimensioni sempre più ampie. Come hanno mostrato Billiet e Dobellaere (1985), il «pilastro» cattolico, termine col quale divenne famosa in Belgio e in Olanda la rete delle organizzazioni confessionali, rimase esternamente intatto (apparendo «cattolico» agli osservatori disinformati), ma l'influenza della Chiesa internamente ad esso diminuì. A vario modo, il risultato finale di questo processo fu la graduale perdita di importanza della religione nella politica dell'Europa occidentale $^{23}$. Dunque, in modo perverso, il cattolicesimo fu prosciugato del suo contenuto religioso anche quando legittimato come identità politica.

La formazione dei partiti confessionali ci mostra quanto fos-

22 L'attenzione dei partiti cristiano-democratici allo stato sociale è, dunque, il risultato di vincoli organizzativi più che ideologici. Se pertanto si vogliono spiegare questi vincoli organizzativi, è necessaria l'analisi del processo di formazione partitica.

23 La «secolarizzazione» dei partiti confessionali è stata, dunque, endogena a questi partiti piuttosto che un adattamento all'ambiente sociale esterno. Lo studio dei partiti confessionali quale fonte della «secolarizzazione» in Europa promette esiti interessanti e stimolanti. 
se complessa, equivoca ed ambigua sin dall'inizio la relazione di questi partiti con la religione e la Chiesa. E dunque sbagliato guardare alla secolarizzazione dei partiti cristiano-democratici come ad un fenomeno del dopoguerra cominciato esogenamente in risposta alla secolarizzazione delle società dell'Europa occidentale. Tale processo ebbe inizio, invece, durante la nascita di quei partiti e fu in larga misura endogeno ad essi, risultato di scelte strategiche compiute sotto i vincoli prodotti dal processo della loro formazione. L'associazione causale di questi partiti con la religione e la Chiesa, nonché la loro riduzione a «istituzioni cattoliche», veicoli delle dottrine della Chiesa o indicatori dell'opinione cattolica, danneggia pertanto la nostra comprensione del fenomeno della democrazia cristiana e della sua influenza politica e sociale. Una corretta comprensione di questi partiti ed una valutazione del loro impatto, estremamente significativo (come indicano le recenti ricerche sociologiche), sebbene fino ad ora trascurato e male interpretato, necessita di una teoria comprensiva della democrazia cristiana che, a sua volta, fornisca importanti elementi al problema più generale della religione e della politica oltre la cristianità e l'Europa.

Sotto il profilo metodologico, questo articolo suggerisce un modo con il quale la teoria della scelta razionale può essere fruttuosamente combinata ad una prospettiva comparata e storica al fine di spiegare fenomeni politici come la formazione partitica. La teoria della scelta razionale impone la specificazione esplicita e rigorosa di assunti sugli attori e sulle preferenze, e richiede una particolare attenzione alle azioni, ai processi, ai meccanismi; l'analisi comparata consente il controllo; e la prospettiva storica, fornendo la «trama» della vicenda, provvede alla conferma empirica sia degli assunti che delle predizioni. La combinazione creativa di approcci apparentemente incompatibili per affrontare un importante problema politico rappresenta, tutto sommato, uno dei percorsi di ricerca più promettenti nella scienza politica.

[Traduzione di Alessandro Chiaramonte]

\section{Riferimenti bibliografici}

Agócs, S. (1988), The Troubled Origins of the Italian Catbolic Labor Movement, 1878-1914, Detroit, Wayne State University Press. 
Anderson, M.L. (1981), Windthorst: A Political Biography, Oxford, Clarendon Press.

- (1986), The Kulturkampf and the Course of German History, in «Central European History», vol. 19, pp. 82-115.

- (1991), Piety and Politics: Recent Work on German Catholicism, in «The Journal of Modern History», vol. 63, pp. 681-716.

Aubert, R. (1975), L'Eglise catbolique de la crise de 1848 à la première guerre mondiale, in R. Aubert, M.D. Knowles e L.J. Rogier (a cura di), Nouvelle bistoire de l'Eglise, Paris, Seuil.

- (1982), La notion de mouvement catbolique. Les enseignements de l'bistoire, in E. De Jonghe e L. Preneel (a cura di), Theorie et language du mouvement catholique. Problèmes d'bistoriographie, Leuven, Universitaire Pers.

Bakvis, H. (1981), Catholic Power in the Netherlands, Kingston-Montreal, McGill University Press.

Bartolini, S. e P. Mair (1990), Identity, Competition, and Electoral Availability: The Stabilization of European Electorates, 1885-1985, Cambridge, Cambridge University Press.

Bessières, A. (1924), Pour l'unité des forces catholique: l'Union Catholique, Paris, J. de Gigord.

Beyme, K. von (1985), Political Parties in Western Democracies, Aldershot, Gower; trad. it. I partiti nelle democrazie occidentali, Bologna, Zanichelli, 1987.

Billiet, J. e K. Dobbelaere (1985), Vers une desinstitutionalisation du pilier chretien?, in L. Voyé, K. Dobbelaere, J. Remy e J. Billiet (a cura di), La Belgique et ses Dieux. Eglises, mouvements religieux et laiques, Louvain-la Neuve, Cabay.

Blackbourn, D. (1991), The Catholic Church in Europe since the French Revolution. A Review Article, in «Comparative Studies in Society and History», vol. 33, pp. 778-790.

Bled, J. (1988), Les fondements du conservatisme autrichien, 1859. 1879, Paris, Publicationes de la Sorbonne.

Bosworth, W. (1962), Catholicism and Crisis in Modern France. French Catholic Groups at the Threshold of the Fifth Republic, Princeton, Princeton University Press.

Boudon, R. (1982), The Unintended Consequences of Social Action, London-Basingstoke, Macmillan.

Bourdieu, P. (1987), What Makes a Social Class? On the Theoretical and Practical Existence of Groups, in «Berkeley Journal of Sociology: A Critical Review», vol. 32, pp. 1-17.

Boutry, P. e A. Michel (1992), La religion, in J. Sirinelli (a cura di), Histoires des droites en France, Paris, Gallimard, vol III.

Boy, D. e N. Meyer (1993), The French Voter Decides, Ann Arbor, The University of Michigan Press.

Boyer, J.W. (1995), Culture and Political Crisis in Vienna. Christian 
Socialism in Power, 1897-1918, Chicago-London, The University of Chicago Press.

Brose, E.D. (1985), Christian Labor and the Politics of Frustration in Imperial Germany, Washington (DC), The Catholic University of America Press.

Broughton, D. (1988), The Social Bases of European Conservative Parties, in B. Girvin (a cura di), The Trasformation of Contemporary Conservatism, London, Sage.

Canavero, A. (1981), Elezioni, in F. Traniello e G. Campanini (a cura di), Dizionario storico del movimento cattolico in Italia, 1860-1980, Genova, Marietti, vol. I, parte II, pp. 285-295.

Castles, F.G. (1994), On Religion and Public Policy: Does Catholicism Make a Difference?, in «European Journal of Political Research», vol. 25, pp. 19-40.

Chapman, G. (1962), The Third Republic of France. The First Phase 1871-1894, New York, St. Martin's Press.

Cholvy, G. (1994), Sociologists, Historians and the Religious Evolution of France from the 18th Century to the Present, in «Modern and Contemporary France», vol. 2, pp. 257-265.

Congar, Y. e F. Varillon (1947), Sacerdoce e laicat dans l'église, Paris, Les Editions du Vitrail.

Dansette, A. (1961), Religious History of Modern France, 2 voll., Freiburg, Herder.

Delbreil, J. (1990), Centrisme et Démocratie-Chrétienne en France. Le Parti Démocrate Populaire des origines au M.R.P. 1919-1944, Paris, Publications de la Sorbonne.

Derivry, D. e M. Dogan (1971), Unité d'analyse et espace de référence en écologie politique. Le canton et le département français, in «Revue Français de Science Politique», vol. 21, pp. 517-570.

De Rosa, G. (1972), Il movimento cattolico in Italia. Dalla Restaurazione all'età giolittiana, Bari, Laterza.

Dondeyne (1964), Priest and Layman, London-New York, Sheed \& Ward.

Durand, J. (1995), L'Europe de la démocratie chrétienne, Bruxelles, Editions Complexe.

Duverger, M. (1966), Sociologie Politique, Paris, Publications Universitaires de France.

Elster, J. (1989), Nuts and Bolts for the Social Sciences, Cambridge, Cambridge University Press; trad. it. Come si studia la società, Bologna, Il Mulino, 1993.

Esping-Andersen, G. (1990), The Three Worlds of Welfare Capitalism, Princeton, Princeton University Press.

Fogarty, M.P. (1957), Christian Democracy in Western Europe, 18201953, London, Routlege \& Kegan Paul.

Formigoni, G. (1988), I cattolici-deputati (1904-1918). Tradizione e riforme, Roma, Edizioni Studium. 
Fox, R.C. (1982), Is Religion Important in Belgium? in «Archives Européenes de Sociologie», vol. 32, pp. 3-38.

Fulton, J. (1987), Religion and Politics in Gramsci: An Introduction, in «Sociological Analysis», vol. 48, pp. 197-216.

Gambasin, A. (1969), Gerarchia e laicato in Italia nel secondo Ottocento, Padova, Antenore.

Geddes, B. (1991), A Game Theoretic Model of Reform in Latin American Democracies, in «American Political Science Review», vol. 85, pp. 371-392.

Gellott, L.S. (1987), The Catholic Church and the Authoritarian Regime in Austria, 1933-1938, New York- London, Garland.

Guyot de Mishaegen, G. (1946), Le Parti Catholique Belge. De 1830 à 1884, Bruxelles, Maison Ferdinand Larcier.

Hanley, D. (1994), Introduction: Christian Democracy as a Political Phenomenon, in D. Hanley (a cura di), Christian Democracy in Europe: A Comparative Perspective, London-New York, Pinter.

Hart, O. (1989), An Economist's Perspective on the Theory of the Firm, in «Columbia Law Review», vol. 89, pp. 1757-1774.

Huard, R. (1982), Le Mouvement Républicain en Bas-Languedoc, Paris, Presses de la Fondation Nationale des Sciences Politiques.

Huber, E., C. Ragin e J.D. Stephens (1993), Social Democracy, Christian Democracy, Constitutional Structure, and the Welfare State, in «American Journal of Sociology», vol. 99, pp. 711-749.

Irvine, W.D. (1989), Royalists, Mass Politics and the Boulanger Affair, in «French History», vol. 3, pp. 31-47.

Irving, R.E.M. (1979), The Christian Democratic Parties of Western Europe, London, Allen \& Unwin [per] il Royal Institute of International Affairs.

Jemolo, A.C. (1960), Church and State in Italy, 1850-1950, Oxford, Basil Blackwell.

Jones, P.M. (1985), Politics and Rural Society: The Southern Massif Central, 1750-1880, Cambridge, Cambridge University Press.

Kalyvas, S.N. (1996), From Pulpit to Party. The Rise of Christian Democracy in Europe, Ithaca-London, Cornell University Press.

Katznelson, I. (1986), Working-Class Formation: Constructing Cases and Comparisons, in I. Katznelson e A.R. Zolberg (a cura di), Working-Class Formation. Nineteenth Century Patterns in Western Europe and the United States, Princeton, Princeton University Press.

Laitin, D. (1986), Hegemony and Culture. Politics and Religious Change Among the Yoruba, Chicago, University of Chicago Press.

Lane, J. e S.O. Ersson (1991), Politics and Society in Western Europe, London, Sage.

La Palombara J. e M. Weiner (1990), The Origin of Political Parties, in P. Mair (a cura di), The West European Party System, Oxford, Oxford University Press. 
Layton-Henry, Z. (1982), Introduction: Conservatism and Conservative Politics, in Z. Layton-Henry (a cura di), Conservative Politics in Western Europe, London-Basingstoke, Macmillan.

Lipset, S.M. e S. Rokkan (1967), Cleavage Structures, Party Systems, and Voter Alignments: An Introduction, in S.M. Lipset e S. Rokkan (a cura di), Party Systems and Voter Alignements: Cross-National Perspectives, New York, The Free Press.

Lönne, K. (1986), Politischer Katholizismus im 19. und 20. Jabrhundert, Frankfurt, Suhrkamp.

Lorwin, V. (1971), Segmented Pluralism. Ideological Cleavages and Political Cohesion in the Smaller European Democracies, in «Comparative Politics», vol. 3, pp. 141-175.

Luebbert, G.M. (1991), Liberalism, Fascism, or Social Democracy. Social Classes and the Political Origins of Regimes in Interwar Europe, New York-Oxford, Oxford University Press.

Lynch, E.A. (1993), Latin America's Christian Democratic Parties. A Political Economy, Westport, Praeger.

Lyon, M. (1967), Christian-Democratic Parties and Politics, in «Contemporary History», vol. 2, pp. 69-89.

Maier, H. (1969), Revolution and the Church. The Early History of Christian Democracy, 1789-1907, Notre Dame, University of Notre Dame Press.

Malgeri, F. (1969), Gli atti dei congressi del Partito Popolare Italiano, Brescia, Morcelliana.

Mann, G. (1968), The History of Germany since 1789, New YorkWashington, Praeger.

May, A.R. (1973), The Falloux Law, the Catholics Press, and the Bishops: Crisis of Authority in the French Church, in «French Historical Studies», vol. VIII, pp. 77-94.

Mayeur, J. (1973), Les débuts de la III République, 1871-1898, Paris, Seuil.

- (1980), Des partis catholiques à la démocratie chrétienne, Paris, Armand Colin.

McLeod, H. (1986), Building the "Catholic Ghetto»: Catholic Organizations 1870-1914, in W. J. Sheils e D. Wood (a cura di), Voluntary Religion, Oxford, Basil Blackwell.

McManners, J. (1972), Church and State in France, 1870-1914, London, SPCK.

Misra, J. e A. Hicks (1994), Catholicism and Unionization in Affluent Postwar Democracies: Catholicism, Culture, Party, and Unionization, in «American Sociological Review», vol. 59, pp. 304-326.

Molony, J. (1977), The Emergence of Political Catholicism in Italy: Partito Popolare, 1919-1926, Totowa, Rowan \& Littlefield.

Olson, M. (1971), The Logic of Collective Action: Public Goods and the Theory of Groups, Cambridge, Harvard University Press; trad. 
it. La logica dell'azione collettiva. I beni pubblici e la teoria dei gruppi, Milano, Feltrinelli, 1983.

Pirenne, H. (1932), Histoire de Belgique. De la Révolution de 1830 à la guerre de 1914, Bruxelles, Maurice Lamertin, vol. VII.

Pizzorno, A. (1970), An Introduction to the Theory of Political Partecipation, in «Social Science Information», vol. 9, pp. 29-61.

Poulat, É. (1977), Église contre bourgeoise. Introduction au devenir du catholicisme actuel, Paris, Casterman.

Preneel, L. (1982), Mouvements catholiques et expériences des catholiques en Belgique, in E. de Jonghe e L. Preneel (a cura di), Theorie et Language du Mouvement Catholique. Problèmes d'Historiographie, Leuven, Universitaire Pers.

Przeworski, A. (1986), Some Problems in the Study of the Transition to Democracy, in G. O'Donnell, P. Schmitter e L. Whitehead (a cura di), Transition fron Authoritarian Rule: Comparative Perspectives, Baltimore-London, The Johns Hopkins University Press.

Przeworski, A. e J. Sprague (1986), Paper Stones: A History of Electoral Socialism, Chicago-London, The University of Chicago Press.

Rahner, K. et al. (1968), Obedience and the Church, Washington-Cleveland, Corpus Books.

Reinermann, A.J. (1993), recensione di R. Lille e F. Traniello (a cura di), Il «Kulturkamp» in Italia e nei paesi di lingua tedesca, in «Catholic Historical Review», vol. 79, pp. 761-763.

Riker, W. (1986), The Art of Political Manipulation, New Haven, Yale University Press.

Rivet, A. (1979), Le vie Politique dans le département de la Haute-Loire de 1815 à 1974, Le Puy, Cahiers de Haute-Loire.

Rokkan, S. (1970), Citizens, Elections, Parties. Approaches to the Comparative Study of the Processes of Development, New York, McKay.

Rommen, H. (1950), The State in Catholic Thought. A Treatise in Political Philosophy, St. Louis, Herder.

Rosenstone, S.J. e J.M. Hansen (1993), Mobilization, Participation, and Democracy in America, New York, Macmillan.

Schorske, C. E. (1967), Politics in a New Key: An Austrian Triptych, in «The Journal of Modern History», vol. 39, pp. 343-386.

Sedgwick, A. (1965), The Ralliement in French Politics, 1890-1898, Cambridge, Harvard University Press.

Sewell, W.H. (1992), Three Temporalities: Toward an Eventful Sociolo$g y$, manoscritto inedito.

Soete, J. (1984), L'ultramontanisme et la formation du parti catholique en Belgique de 1875 à 1884, in E. Lamberts (a cura di), De Kruistocht tegen bet Liberalisme: facetten van het ultramontanisme in Belgie in de 19e eeuw, Leuven, Universitaire Pers.

Stengers, J. (1983), Léopold II et le cabinet Frère-Orban (1878-1884). Correspondence entre le roi et ses ministres, 2 voll., Cahiers du 
Centre Interuniversitaire d'Histoire Contemporaine, 95, Leuven, Éditions Nauwelaerts.

Suardo, D.S. (1962), I cattolici intransigenti, Brescia, Morcelliana.

Therborn, G. (1994), Another Way of Taking Religion Seriously. Comment on Francis G. Castles, in «European Journal of Political Science», vol. 26, pp. 103-110.

Traniello, F. (1982), Le mouvement catholique en Italie jusqu'à la première guerre mondiale: problèmes de méthode et de définition, in E. de Jonghe e L. Preneel (a cura di), Theorie et Language du Mouvement Catholique. Problèmes d'Historiographie, Leuven, Universitaire Pers.

Vaillancourt, J.G. (1980), Papal Power. A Study of Vatican Control Over Lay Catholic Elites, Berkeley, University of California Press.

Van Kersbergen, K. (1994), The Distinctiveness of Christian Democracy, in D. Hanley (a cura di), Christian Democracy in Europe: A Comparative Perspective, London-New York, Pinter.

- (1995), Social Capitalism: A Study of Christian Democracy and the Welfare State, London, Routledge and Kegan Paul.

Vecchio, G. (1979a), La democrazia cristiana in Europa (1891-1963), Milano, Mursia.

- (1979b), Interpretation of the Italian Popular Party and the Italian Catbolic Movement, in «The Journal of Italian History», vol. 2, pp. 52-74.

Verdier, D. (1994), Democracy and International Trade. Britain, France, and the United States, 1860-1990, Princeton, Princeton University Press.

Viance, G. (1930), La Fédération nationale catholique, Paris, Ernest Flammarion.

Weber, M. (1963), The Sociology of Religion, Boston, Beacon Press.

Wilensky, H. (1981), Leftism, Catholicism and Democratic Corporatism: The Role of Political Parties in Recent Welfare State Development, in P. Flora e A.J. Heidenheimer (a cura di), The Development of Welfare States in Europe and America, New Brunswick, Transaction Books.

Windell, G.G. (1954), The Catholics and German Unity, 1866-1871, Minneapolis, University of Minnesota Press.

Woeste, C. (1927), Mémoires pour servir à l'Histoire contemporaine de la Belgique, Bruxelles, Librairie Albert Dewit, vol. I.

Zeps, M.J. (1979), The Politics of Education in Austria: Church, State and the Reform of Education, 1765-1962, tesi di dottorato, Stanford University.

Zerbi, P. (1961), Il Movimento Cattolico in Italia da Pio IX a Pio X. Linee di sviluppo, Milano, Vita e Pensiero. 